\title{
Fractional-order two-component oscillator: stability and network synchronization using a reduced number of control signals
}

\author{
Romanic Kengne $^{1,2}$, Robert Tchitnga ${ }^{1,2,3}$, Alain Kammogne Soup Tewa ${ }^{2}$, Grzegorz Litak ${ }^{4,5, a}$, \\ Anaclet Fomethe ${ }^{6}$, and Chunlai $\mathrm{Li}^{7}$ \\ ${ }^{1}$ Research Group on Experimental and Applied Physics for Sustainable Development, Faculty of Science, \\ Department of Physics, University of Dschang, P.O. Box 412, Dschang, Cameroon \\ ${ }^{2}$ Laboratory of Electronics and Signal Processing Faculty of Science, Department of Physics, University of Dschang, \\ P.O. Box 67, Dschang, Cameroon \\ ${ }^{3}$ Institute of Surface Chemistry and Catalysis, University of Ulm, Albert-Einstein-Allee 47, 89081 Ulm, Germany \\ ${ }^{4}$ Lublin University of Technology, Faculty of Mechanical Engineering, Nadbystrzycka 36, 20-618 Lublin, Poland \\ ${ }^{5}$ AGH University of Science and Technology, Faculty of Mechanical Engineering and Robotics, Department of \\ Process Control, Mickiewicza 30, 30-059 Krakow, Poland \\ ${ }^{6}$ Laboratoire de Mécanique et de Modélisation des Systèmes, L2MS, Department of Mathematics and Computer \\ Science, Faculty of Science, University of Dschang, P.O. Box 67, Dschang, Cameroon \\ 7 College of Physics and Electronics, Hunan Institute of Science and Technology Yueyang, Hunan 414006, \\ P.R. China
}

Received 30 May 2018 / Received in final form 20 August 2018

Published online 5 December 2018

(C) The Author(s) 2018. This article is published with open access at Springerlink.com

\begin{abstract}
In this paper, a fractional-order version of a chaotic circuit made simply of two non-idealized components operating at high frequency is presented. The fractional-order version of the Hopf bifurcation is found when the bias voltage source and the fractional-order of the system increase. Using AdamsBashforth-Moulton predictor-corrector scheme, dynamic behaviors are displayed in two complementary types of stability diagrams, namely the two-parameter Lyapunov exponents and the isospike diagrams. The latest being a more fruitful type of stability diagrams based on counting the number of spikes contained in one period of the periodic oscillations. These two complementary types of stability diagrams are reported for the first time in the fractional-order dynamical systems. Furthermore, a new fractional-order adaptive sliding mode controller using a reduced number of control signals was built for the stabilization of a fractional-order complex dynamical network. Two examples are shown on a fractional-order complex dynamical network where the nodes are made of fractional-order two-component circuits. Firstly, we consider an ideal channel, and secondly, a non ideal one. In each case, increasing of the coupling strength leads to the phase transition in the fractional-order complex network.
\end{abstract}

\section{Introduction}

Fractional calculus is a more than 300-years-old development in the mathematics since it has been introduced by Leibniz [1]. Its application in various fields of science and engineering has yield to its recognition as an efficient tool to describe with more accuracy models encountered in integer-order systems [2]. In the past, scientists were longing to use fractional differential equations due to lack of methods for solving them. Nowadays, several techniques reported in the literature are used to approximate the fractional derivatives, as well as fractional integrals [1-7]. Therefore, in recent years its applications to physics and engineering received a great attention. It was found that many real dynamical systems and processes in interdisciplinary fields can be described by fractional

\footnotetext{
${ }^{\mathrm{a}}$ e-mail: g.litak@pollub.pl
}

differential equations. Diffusion of heat into a semiinfinite solid [1], voltage current relation of a semi-infinite lossy transmission line [8], viscoelastic systems [9], dielectric polarization [3], magnetization [10], as well a modeling love between humans [11] happiness [12], nonlocal epidemics [13], El Niño phenomenon [14], logistic delay systems [15] or actively controlling chaos in economics [16] are just few examples of fractional-order derivative applications. Recently, many researchers started showing great interest in a chaotic behavior of fractional-order dynamical systems. The Caputo fractional order derivative allows to describe dynamical systems more accurately than their integer order counterparts $[17,18]$. Therefore, several fractional order generic systems were proposed starting from the chaotic integer-order counterparts. For example, by considering the pioneering Chua's circuit [19], some fractional-order counterparts have been proposed [20-22]. Paper [23] proposed a systematic design approach 
for creating multi-wing chaotic attractors in an uncoupled fractional-order differential system. Li and Chen [24] found that chaos exists in the fractional-order Chen system with order less than three. In [25], based on the Grünwald-Letnikov method, Kengne et al. investigated the dynamics of a fractional-order two-stage Colpitts oscillator. Donato and Giuseppe, in reference [26], presented as the simplest fractional-order memristor based chaotic system. Starting from the integer-order counterpart, a detailed and well illustrated theoretical analysis of its dynamics, along with accurate numerical simulations showing chaotic behaviors and oscillations, were provided. It is worth to notify, that all the chaotic systems presented so far, are operated at low frequency. Namely, it is somehow a real drawback for some electronic applications. Meanwhile, in [27] it is shown that electronic circuits which operate at high frequency and having a high degree of freedom are of great advantage in cryptography and can contribute to the generation of random or pseudo-random bit streams with much reasonable speeds, and at low cost. In [28] Tchitnga et al. proposed the simplest chaotic two-component Hartley circuit. This circuit operating at high frequency may be a good candidate for the cryptography and for the generation of random bit streams. In [27], Nguimdo et al. increased the complexity of the simplest and low-cost chaotic system by coupling three such circuits, with the goal to test their application for random bit generation. Recently, two complementary types of diagrams were presented in order to characterize the stability of dynamic systems, namely: the stability diagram based on twoparameters Lyapunov exponents and Isospike diagram based on counting the number of spikes contained in one period of the periodic oscillations [29-35]. In [29] based on the two-parameters Lyapunov exponents and isospike diagram, the authors report numerically computed stability phase diagrams indicating precisely where spirals of periodicity and chaos may be found in several control planes of simplest two-component Hartley-like circuit. To the best of our knowledge, no study has been proposed in the literature on the stability of fractional order systems based on isospike diagrams. Recently, synchronization of chaotic fractional differential systems started to attract increasing attention due to its potential applications in secure communication and control processing [36-39]. In fact, with the memory effects and history dependence that the fractional order brings, the control systems have the possibility to use all of the past information for control and decision [40,41]. Concluding the main results obtained through synchronization between two chaotic fractional order systems, it is worth to mention that the modified projective synchronization of fractional order chaotic systems was investigated in [42]. While the hybrid projective synchronization of a fractional order chaotic system was presented in $[43,44]$. In [45] based on the adaptive open-plus-closed-loop method, a general method of function projective synchronization was derived, where its robustness was tested with effects against noise. Outer synchronization between two different fractional-order general complex dynamical networks was investigated in [46]. In [47], based on stability criteria of fractionalorder system, the authors achieved synchronization of
$\mathrm{N}$-coupled fractional-order chaotic systems with unidirectional coupling and bidirectional coupling. Pinning control and synchronization of complex networks with coupling terms were studied due to its theoretical importance and practical application [48-52]. In these works, firstly the authors used all the directions of the node to insure the synchronization of complex networks which is a very drawback of the practical view point [53], secondly these authors used the usual form of the adaptive feedback control law which is not robust for the synchronization in presence of perturbation and the uncertainties. Unfortunately, these works did not present any stability discussion for the sliding mode dynamics. On the other hand, the sliding mode controller has some attractive advantages, including: fast dynamic responses and good transient performance, external disturbance rejection, and insensitivity to parameter variations and model uncertainties $[54,55]$. The advantage to use an adaptive sliding mode controller incorporating a fractional order derivative is twofold [56,57]: (i) the fractional order derivative improves the control performance compared to the traditional integer order derivative because of the increase of degrees of freedom of systems; (ii) the adaptive law improves the robustness of the synchronization scheme.

At best of knowledge, few authors used the controller integrating the sliding mode for the synchronization of fractional-order complex network [58,59]. Furthermore, very few works contributed to the synchronization of the complex network have interest in the phase-transition [49-52]. In this paper our contribution consist firstly to proposed the fractional-order version of two-parameters Lyapunov exponents diagram and the isospike diagram applied to fractional-order two-component Hartley-like circuit. Secondly, we build a novel controller for the robust generalized adaptive sliding mode synchronization of the fractional-order complex network and our controller using a single-state to realize the asymptotical stability of synchronization error system. Two examples are given on the case of fractional-order two-component chaotic circuit and in each of the example the phase-transition is shown when the coupling strength increases. In the first example, we consider the ideal channel of transmission, and in the second example we consider the non-ideal channel of transmission between the isolated node (master) and the nodes of fractional-order complex network. The rest of this paper is organized as follows: in Section 2, the fractional-order two-component circuit is proposed and its dynamics studies. Section 3 deals, with the adaptive feedback synchronization of the fractional-order complex network and an example is applied on the fractional-order complex network of the two-component circuit; numerical simulations are given to sustain the theoretical analysis. The general conclusion constitute the object of Section 4 .

\section{The fractional-order version of a two-component simplest chaotic circuit}

\subsection{Equations model}

Consider the circuit given in [28] and described by Figure 1, the fractional-order of this circuit in dynamics 


$$
I_{\mathrm{d}}=\left\{\begin{array}{lcc}
0 & \text { if } & V_{\mathrm{GS}} \leq V_{\mathrm{GSoff}} \\
g_{m 0}\left(V_{\mathrm{GS}}-V_{\mathrm{GSoff}}\right)^{2} & \text { if } & V_{\mathrm{GD}} \leq V_{\mathrm{GSoff}} \\
g_{m 0}\left(V_{\mathrm{GS}}-V_{\mathrm{GD}}\right)\left(V_{\mathrm{GS}}+V_{\mathrm{GD}}-2 V_{\mathrm{GSoff}}\right) & \text { if } & V_{\mathrm{GD}} \geq V_{\mathrm{GSoff}}
\end{array}\right.
$$

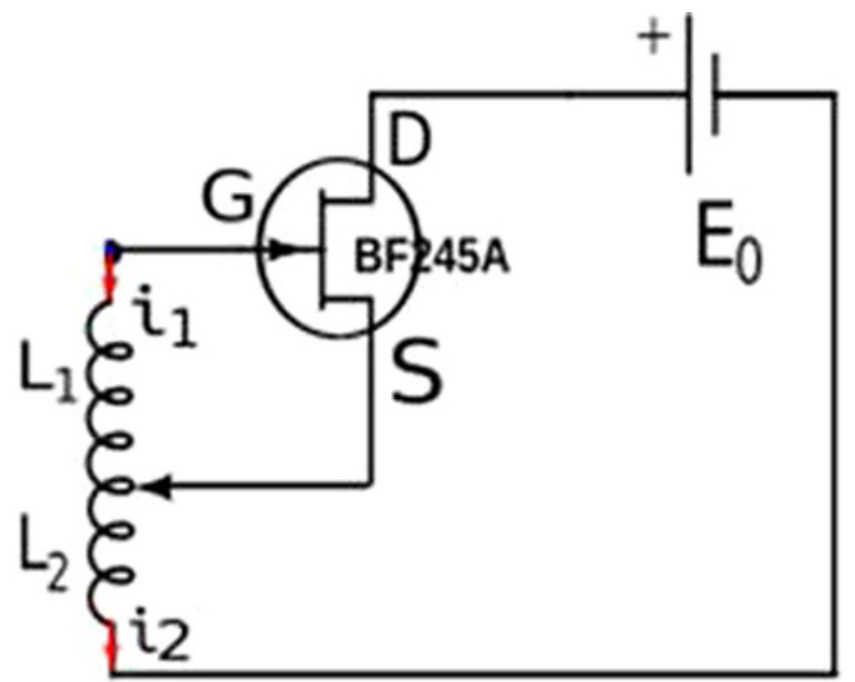

Fig. 1. Circuit model of the two-component Hartley's oscillator using a JFET and a tapped coil with the upper inductance $L 1$ and lower inductance $L 2$.

regime using the simplified Giacoletto dynamic model of the junction field effect transistor (JFET) [28] for small signals at high frequency is represented on Figure 2. In this circuit we suppose that through the parasitic capacitors represented by the capacitances $C_{\mathrm{GS}}$ and $C_{\mathrm{GD}}$ are flowing currents which are respectively function of the fractional-order derivative $q_{1}$ and $q_{2}$, and expressed by $i_{C_{\mathrm{GS}}}=C_{\mathrm{GS}} \frac{d^{q_{1}} V_{\mathrm{GS}}}{d t^{q_{1}}}$ and $i_{C_{\mathrm{GD}}}=C_{\mathrm{GD}} \frac{d^{q_{2}} V_{\mathrm{GD}}}{d t^{q_{2}}}$. The voltages across the fractional-order inductors parts $L_{1}, L_{2}$, namely $V_{L_{1}}=L_{1} \frac{d^{q_{3} i_{1}}}{d t^{q_{3}}}$ and $V_{L_{2}}=L_{2} \frac{d^{q_{4}} i_{2}}{d t^{q_{4}}}$ are function respectively of the fractional-order derivatives $q_{3}$ and $q_{4}$. The two nonlinearities in this circuit are provided by the internal current source of the JFET (Eq. (1)) and the internal diode at the gate-source (GS) junction (Eq. (2)):

\section{See equation (1) above}

and

$$
i_{\mathrm{D}}=I_{\mathrm{S}}\left[\exp \left(\frac{V_{\mathrm{GS}}}{V_{T}}\right)-1\right] .
$$

The three lines of equation (1) depict the cutoff region of the JFET, the saturation and the triode regions respectively. Note that $V_{\mathrm{GSoff}} \leq 0$ is the cutoff value of the voltage between the gate and the source of the JFET; $g_{m 0}$ is its current gain; $E$ is the bias voltage source. The application of the Kirchhoff laws on the circuit (Fig. 2) enables to describe the dynamics of the system through the following set of equations:

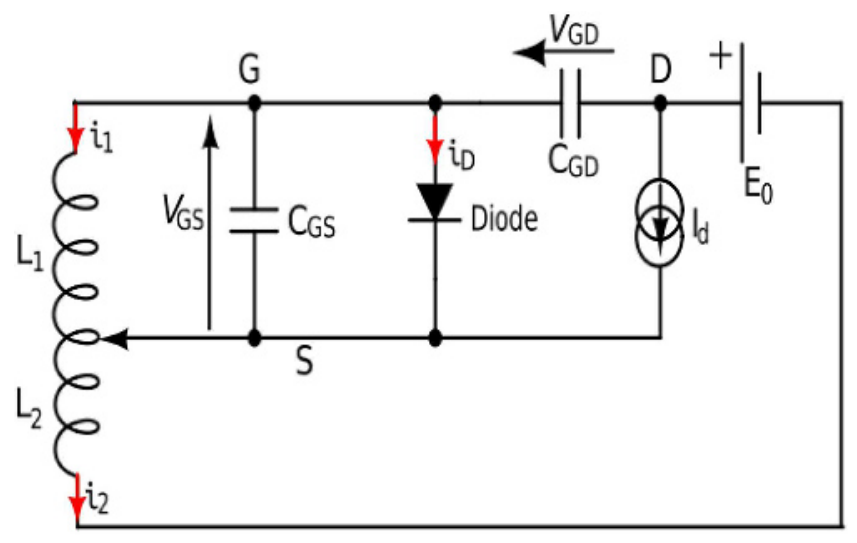

Fig. 2. Equivalent circuit of two-component Hartley's oscillator using the simplified Giacoletto dynamic model of the JFET for small signals at high frequency.

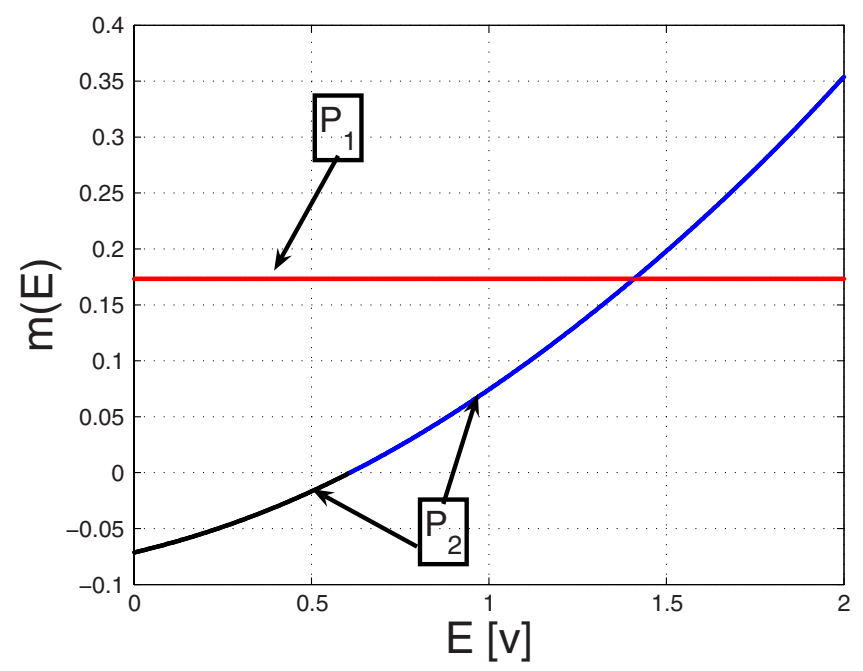

Fig. 3. Representation of values of $m(E)=q \pi / 2-$ $\min \left|\arg \left(\lambda_{i}\right)\right|$ around of the two fixes points $P_{1}$ (red curve) and $P_{2}$ (black and blue curve) when the bias voltage source is increases, and $q=0.96$.

$$
\left\{\begin{array}{l}
\frac{d^{q_{1}} V_{\mathrm{GS}}}{d t^{q_{1}}}=\frac{1}{C_{\mathrm{GS}}}\left(-i_{1}+i_{2}-i_{\mathrm{D}}-I_{d}\right) \\
\frac{d^{q_{2}} V_{\mathrm{GD}}}{d t^{q_{2}}}=\frac{1}{C_{\mathrm{GD}}}\left(-i_{2}+I_{d}\right) \\
\frac{d^{q_{3}} i_{1}}{d t^{q_{3}}}=\frac{1}{L_{1}} V_{\mathrm{GS}} \\
\frac{d^{q_{4}} i_{2}}{d t^{q_{4}}}=\frac{1}{L_{2}}\left(-V_{\mathrm{GS}}+V_{\mathrm{GD}}+E\right) .
\end{array}\right.
$$

The variables $i_{1}$ and $i_{2}$ denote the current flowing through the inductor parts $L_{1}$ and $L_{2}$ respectively, while $i_{D}$ is the nonlinear current defined by (Eq. (7)). In the 


$$
M_{\mathrm{J}_{2}}=\left(\begin{array}{cccc}
-\frac{I_{\mathrm{S}}}{C_{\mathrm{GS}} V_{T}}+\frac{2 g_{m 0} V_{\mathrm{GSoff}}}{C_{\mathrm{GS}}} & -2 \frac{g_{m 0}}{C_{\mathrm{GS}}}\left(E+V_{\mathrm{GSoff}}\right) & -\frac{1}{C_{\mathrm{GS}}} & \frac{1}{C_{\mathrm{GS}}} \\
-2 \frac{g_{m 0}}{C_{\mathrm{GD}}} V_{\mathrm{GSoff}} & 2 \frac{g_{m 0}}{C_{\mathrm{GD}}}\left(E+V_{\mathrm{GSoff}}\right) & 0 & -\frac{1}{C_{\mathrm{GD}}} \\
\frac{1}{L_{1}} & 0 & 0 & 0 \\
-\frac{1}{L_{2}} & \frac{1}{L_{2}} & 0 & 0
\end{array}\right)
$$

rest of the paper, following values of parameters are going to be considered, which have been used for the simulation aspect of the work via the virtual electronic laboratory PSpice: $C_{\mathrm{GS}}=3.736 \mathrm{pf}, C_{\mathrm{GD}}=3.35 \mathrm{pf}, L_{1}=$ $24.5 \mu \mathrm{H}, L_{2}=4 \mu \mathrm{H}, I_{\mathrm{S}}=33.57 \mathrm{fA}, V_{\mathrm{GSoff}}=-1.409 \mathrm{~V}$, $g_{m 0}=1.754 \mathrm{mAV}^{-2}, V_{\mathrm{T}}=25 \mathrm{mV}$.

\subsection{Equilibrium points and stability}

The equilibrium points of the fractional-order two component system (3) are as follows: $P_{1}\left(0,-E, 0, g_{m 0} V_{\mathrm{GSoff}}^{2}\right)$ in the saturation region, $P_{2}\left(0,-E, 0,-g_{m 0} E\left(E+2 V_{\mathrm{GSoff}}\right)\right)$ in the triode region. In the cutoff region the system does not have an equilibrium point, due to the fact that the condition on the cutoff value of the voltage between the GS electrodes does not allow it.

The Jacobian matrix around the fixed point, in the saturation region is:

$M_{\mathrm{J}_{1}}=\left(\begin{array}{cccc}-\frac{I_{\mathrm{S}}}{C_{\mathrm{GS}} V_{\mathrm{T}}}+\frac{2 g_{m 0} V_{\mathrm{GSoff}}}{C_{\mathrm{GS}}} & 0 & -\frac{1}{C_{\mathrm{GS}}} & \frac{1}{C_{\mathrm{GS}}} \\ -\frac{2 g_{m 0} V_{\mathrm{GSoff}}}{C_{\mathrm{GD}}} & 0 & 0 & -\frac{1}{C_{\mathrm{GD}}} \\ \frac{1}{L_{1}} & 0 & 0 & 0 \\ -\frac{1}{L_{2}} & \frac{1}{L_{2}} & 0 & 0\end{array}\right)$,

and the Jacobian matrix around the fixed point $P_{2}$ in the triode region is:

\section{See equation above.}

Setting: $\alpha=\frac{C_{\mathrm{GS}}}{C_{\mathrm{GD}}}, a_{1}=\frac{1}{C_{\mathrm{GS}}}, a_{2}=\frac{I_{\mathrm{S}}}{C_{\mathrm{GS}} V_{\mathrm{T}}}, a_{3}=\frac{g_{m 0}}{C_{\mathrm{GS}}}, b_{1}=$ $\frac{1}{L_{1}}, b_{2}=\frac{1}{L_{2}}, x_{m}=V_{\mathrm{GSoff}}$. The characteristic equation solution of $\operatorname{det}\left(\operatorname{diag}(\lambda)-M_{\mathrm{J}_{1}}\right)=0$ at $P_{1}$ becomes:

$$
\begin{aligned}
& \lambda^{4}+\left(a_{2}-2 a_{3} x_{m}\right) \lambda^{3}+a_{1}\left(\alpha b_{2}+b_{2}+b_{1}\right) \lambda^{2}+\alpha a_{1} a_{2} \lambda \\
& \quad+\alpha a_{1}^{2} b_{1} b_{2}=0,
\end{aligned}
$$

while at $P_{2}$, that characteristic equation solution of $\operatorname{det}\left(\operatorname{diag}(\lambda)-M_{J_{2}}\right)=0$ is:

$$
\begin{aligned}
\lambda^{4} & +\left(a_{2}-2 \alpha a_{3} e-2 a_{3} x_{m}-2 \alpha a_{3} x_{m}\right) \lambda^{3} \\
& +\left(a_{1} b_{1}+a_{1} b_{2}+\alpha a_{1} b_{2}-2 \alpha a_{2} a_{3} x_{m}-2 \alpha a_{2} a_{3} e\right) \lambda^{2} \\
& \quad-\alpha a_{1}\left(2 a_{3} b_{1} x_{m}+2 a_{3} b_{1} e-a_{2} b_{2}\right) \lambda^{1}+\alpha a_{1}^{2} b_{1} b_{2}=0 .
\end{aligned}
$$

Theorem 1. [58] The following commensurate order system:

$$
{ }_{t_{0}}^{C} D_{t}^{q} X(t)=A X(t), X(0)=X_{0},
$$

with $0<q \leq 1, R^{n}$, and $A \in R^{n}$ is asymptotically stable if and only if $m=q \pi / 2-\min \left|\arg \left(\lambda_{i}\right)\right|<0 \quad(1 \leq i \leq n)$ is satisfied for all eigenvalues $\lambda_{i}$ of the matrix A. Moreover, this system is stable if and only if $m=q \pi / 2-\min \left|\arg \left(\lambda_{i}\right)\right| \leq 0 \quad(1 \leq i \leq n)$ is satisfied for all eigenvalues $\lambda_{i}$ of $A$ with those of the critical eigenvalues that satisfy $m=q \pi / 2-\min \left|\arg \left(\lambda_{i}\right)\right|=0 \quad(1 \leq i \leq n)$ having geometric multiplicity of one.

The stability of the system around its equilibrium points $P_{1}$ and $P_{2}$ in the space $(q, E)$ is appreciable on Figure 3 . Here the bias voltage source $E$ is used as control parameter for the stability study. Taking advantage of Theorem 1 and based on the two characteristics equations, Theorem $m(E)=q \pi / 2-\min \left|\arg \left(\lambda_{i}\right)\right|$ is plotted. It can be observed that the red curve depicts $m(E)$ around the fixed point $P_{1}$ and the black and blue curves are the plot of the same function around the next fixed point $P_{2}$. The red curve reveals that, for any value of the control parameter $E$, the function value of $m(E)>0$, and no change of its sign. Using the Theorem 1 , it can be therefore be deduced that the system is unstable around the equilibrium point $P_{1}$. The black and blue curves on the other hand reveal that, in the first interval $0<E<0.6$, $m(E)<0$ which traduces the local stability of the system around $P_{2}$, while in the second interval $0.6<E<2$, $m(E)>0$, meaning that the system is instable around the equilibrium point $P_{2}$. This last result shows that the system changes its state in the neighborhood of $E=0.6 \mathrm{~V}$. For this reason, it is important to study the bifurcations around the equilibrium point $P_{2}$ as a function of the control parameter $E$.

\subsection{The Hopf bifurcation}

Let us investigate the numerical Hopf bifurcation in the fractional-order two-component oscillator. Three conditions listed below are necessary to see the fractional-order system (Eq. (3)) undergo a Hopf bifurcation at its adequate equilibrium point, when the bifurcation parameters $(q, E)$ take the critical values $q^{*} \in(0,1)$ and $E^{*}$ :

- the corresponding characteristic equation (7) must have a pair of complex conjugate roots 

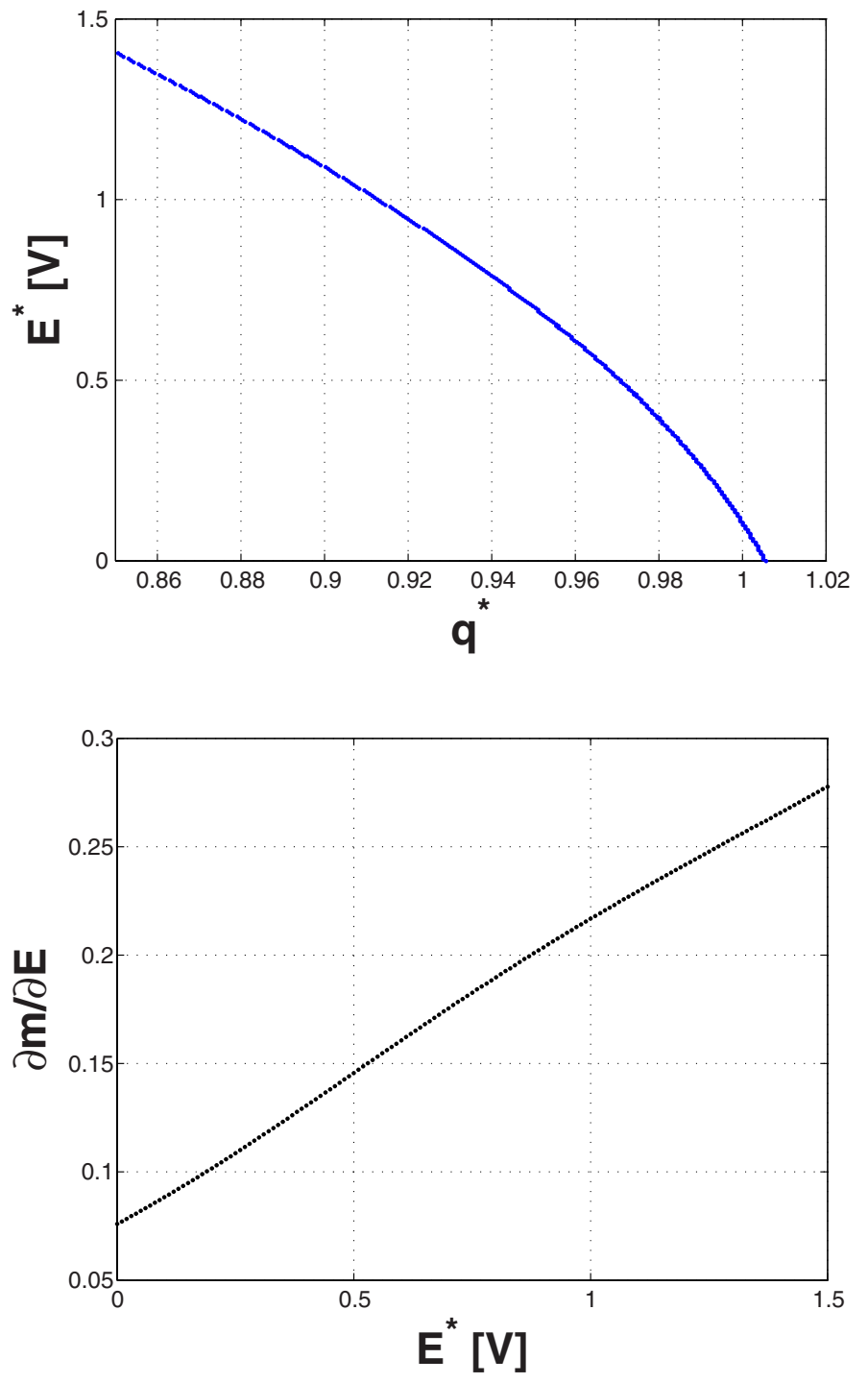

Fig. 4. Critical values $E^{*}$ versus the fractional order $q^{*}$. (a) This curve depicts the couples of values for which the Hopf bifurcation occurs in the system and evolution of $\frac{d m(q, E)}{d E}$ versus $E^{*}$ on (b).

$$
\begin{aligned}
& \lambda_{1,2}\left(E^{*}\right)=\alpha\left(E^{*}\right) \pm i \beta\left(E^{*}\right)\left(\alpha\left(E^{*}\right)>0\right), \text { and two } \\
& \text { negatives reals roots } \lambda_{3} \text { and } \lambda_{4} ; \\
- & m\left(q^{*}, E^{*}\right)=q^{*} \pi / 2-\min \left|\arg \left(\lambda_{i}\right)\right|=0 ; \\
- & \left.\frac{d m(q, E)}{d q}\right|_{q=q^{*}} \neq 0 \text { and }\left.\frac{d m(q, E)}{d E}\right|_{E=E^{*}} \neq 0 \text { (transver- } \\
& \text { sality conditions). }
\end{aligned}
$$

Figure $4 \mathrm{a}$ depicts the solutions $\left(q^{*}, E^{*}\right)$ of equation $m(q, E)=0$, while Figure $4 \mathrm{~b}$ recalls the two transversality conditions $\left.\frac{\mathrm{d} m(q, E)}{d E}\right|_{E=E^{*}} \neq 0$ and $\left.\frac{d m(q, E)}{d q}\right|_{q=q^{*}}=\frac{\pi}{2} \neq 0$ which lead to the conclusion that for any couple $\left(q^{*}, E^{*}\right)$ of critical values, system (7) undergoes the Hopf bifurcation around the fixed point $P_{2}$, confirming therefore what could already be assumed from the previous subsection.

\subsection{Stability diagrams}

The phase diagrams characterizing the far-reaching regular organization induced by the set of stable oscillations of the fractional-order two-component circuit is presented in two complementary ways in Figure 5. The numerical approach for solving fractional-order differential equation (3) is that of Adams-Bashforth-Moulton predictor-corrector scheme with fixed time-step $h=5 \times 10^{-11}$. This approach is based on the approximative Caputo method of the fractional-order derivative, given by:

$$
\begin{aligned}
{ }_{t_{0}}^{C} D_{t}^{q} w(t) & ={ }_{t_{0}}^{C} D_{t}^{-(k-q)} \frac{d^{k}}{d t^{k}} w(t) \\
& =\frac{1}{\Gamma(k-q)} \int_{t_{0}}^{t}(t-\tau)^{k-q-1} w^{(k)}(\tau) d \tau
\end{aligned}
$$

where $k-1<q<k \in \mathbb{Z}^{+}$.

The detailed description of this algorithm is available in reference [4]. To produce the diagrams, a selected parameter window of interest is covered with a mesh of $150 \times 150$ equidistant points. For all these diagrams, the fractional-order integrations were started always from the same initial conditions: $V_{\mathrm{GS}}=-1.25 \mathrm{~V}, V_{\mathrm{GD}}=-2.5 \mathrm{~V}$, $i_{1}=1 \mu \mathrm{A}$ and $i_{2}=1 \mu \mathrm{A}$. The first $0.3 \times 10^{5}$ integration steps were unheeded as transient time needed to approach the solution with the subsequent $10^{5}$ steps used to compute the largest Lyapunov spectrum and get the number of peaks (maxima) contained in one period of solution. Estimation of the largest Lyapunov exponnent were performed with ignoring the fractional derivative effect on the embeding dimension. This simplified algorithm was presented by Syta et al. [60]. This led us to obtain the isospike diagrams presented in Figure 5. For more details on the description of isospike diagram algorithms see references [29-35].

The Lyapunov stability diagrams of fractional-order two-component circuit presented on Figures $5 \mathrm{a}-5 \mathrm{c}$ discriminate the chaotic motion (positive exponents) from the periodic nature (negative exponents) of oscillations in the $q \times E, L_{1} \times L_{2}$, and $C_{\mathrm{GS}} \times C_{\mathrm{GD}}$ parameters' plans respectively. The values of parameters used to plot the three panels are listed in Section 2.1. It is worth mentioning that for the last two plans $L_{1} \times L_{2}$ and $C_{\mathrm{GS}} \times C_{\mathrm{GD}}$ the following parameters have been kept constant at $q=0.98$ and $E=3 \mathrm{~V}$. Notice that the Lyapunov exponent presented here is a qualitative largest Lyapunov exponent. Different Lyapunov consistencies have been distinguished by colors: red for the negative Lyapunov exponents, yellow for the low-largest Lyapunov exponents and finally black for the high largest Lyapunov exponent. To each Lyapunov exponent figure (Figs. 5a-5c) corresponds an isospike diagram (Figs. 5d-5f) respectively. Each isospike figure is a superposition of 1-17 spikes in one period of regular oscillations. The three isospikes diagrams depict different mathematical phenomena.

Figure 5d representing the isospike diagram of $E$ as a function of $q$, has been obtained using a palette of nine colors for regular oscillations in one period of the 

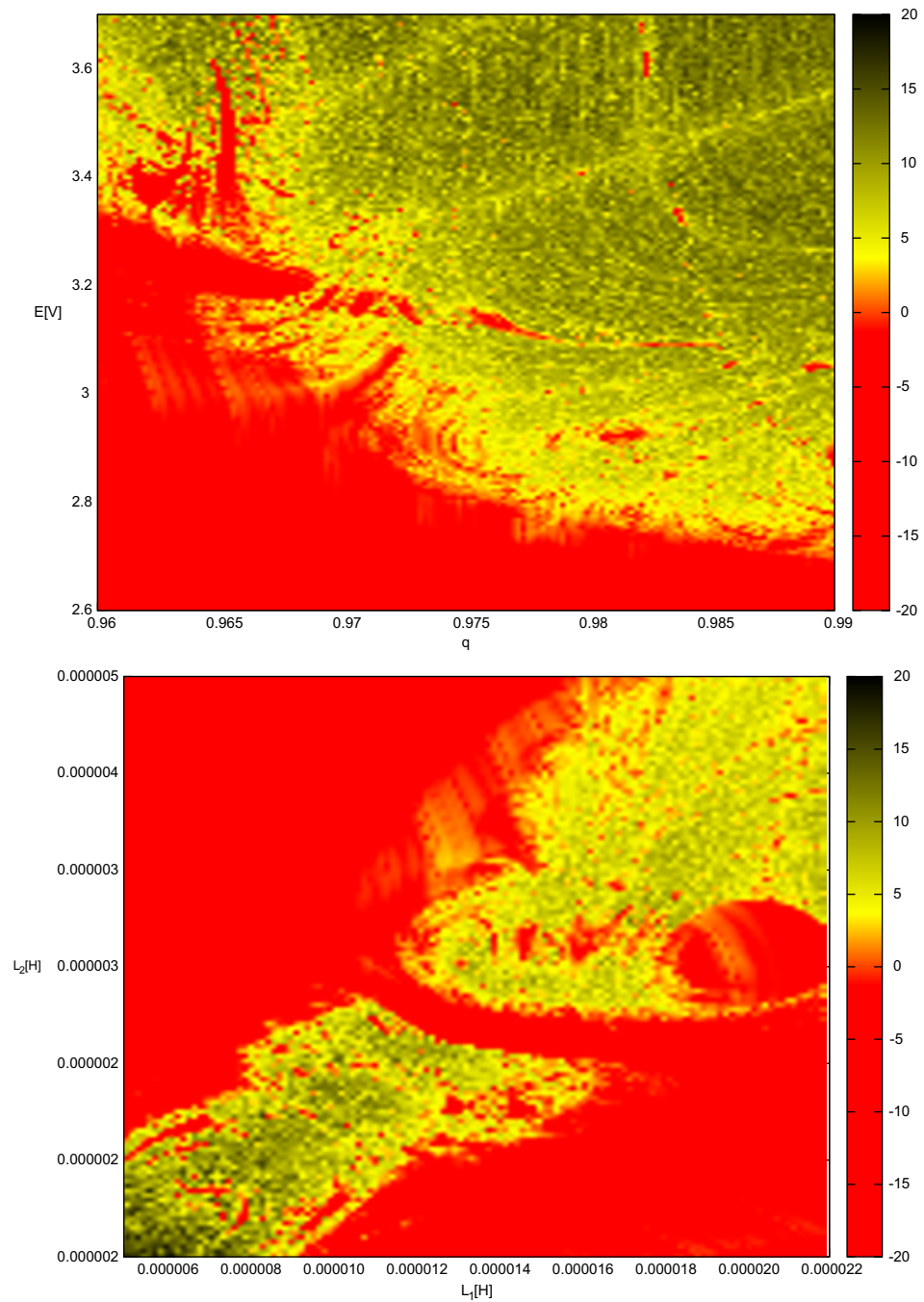

(b)

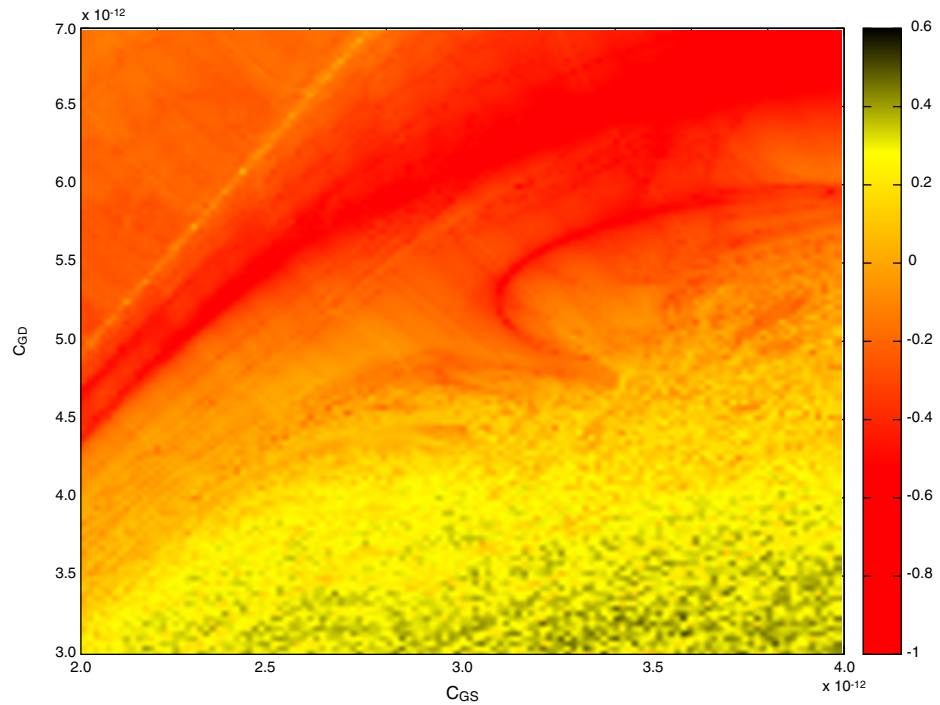

(c)

Fig. 5. Two complementary ways of characterizing the stability of the circuit in the $q \times E, L_{1} \times L_{2}$, and $C_{\mathrm{GS}} \times C_{\mathrm{GD}}$ control planes respectively: $(\mathrm{a}-\mathrm{c})$ Lyapunov diagrams, and $(\mathrm{d}-\mathrm{f})$ isospike diagrams displaying the number of peaks in one period of $V_{\mathrm{GS}}$ $[(\mathrm{d}-\mathrm{e})]$ and in one period of $V_{\mathrm{GD}}(\mathrm{f})$. Absence of periodicity (chaos) is represented in black. The regions in white color are regions of quasi-periodicity. In (a), (d), the parameters are chosen as in Section 2.1, while in (b), (c), (e), (f) the rest of parameters are: $q=0.98$ and $E=3 \mathrm{~V}$. Each panel shows $150 \times 150=22500$ parameters points. 

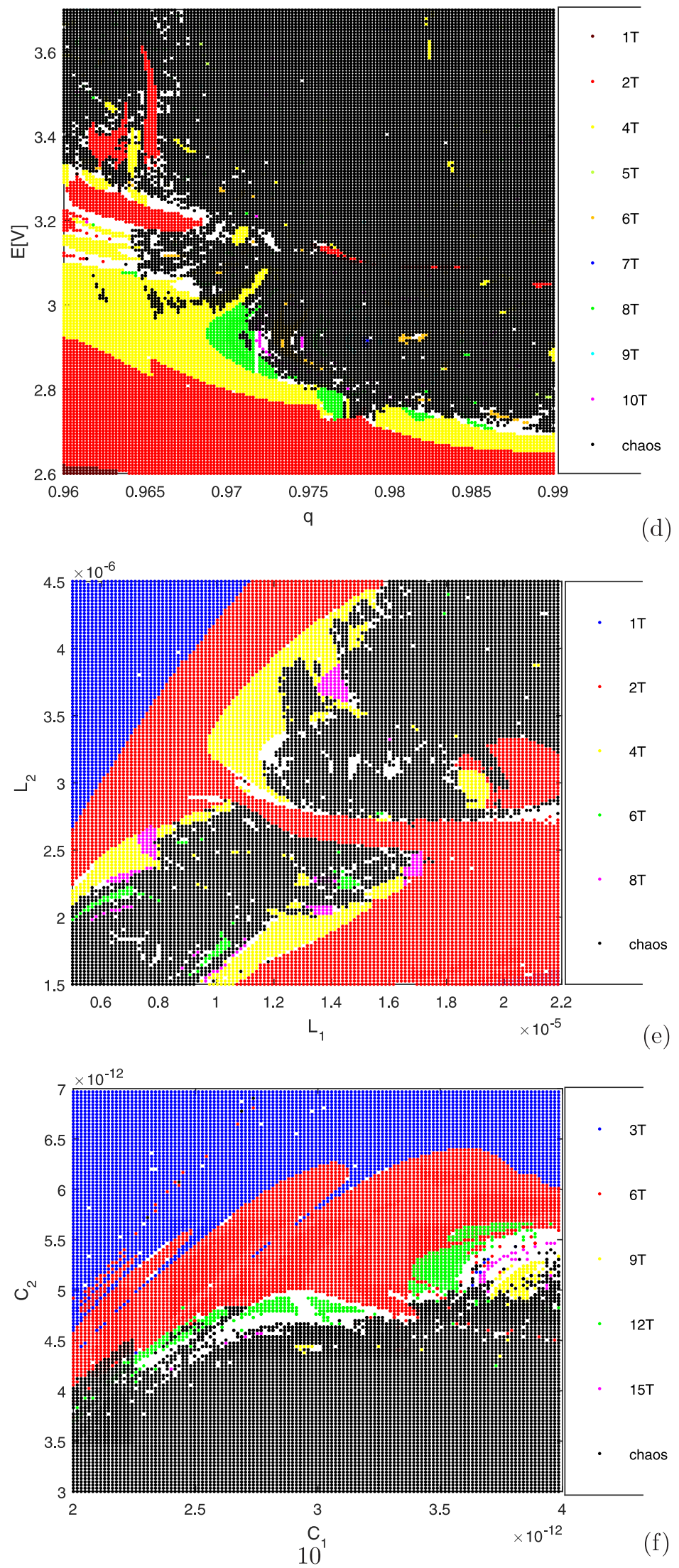

Fig. 5. (Continued) 
Table 1. Number of peaks in one period of oscillations of $V_{\mathrm{GS}}$ as a function of the fractional-order $q$ and bias voltage source $E$.

\begin{tabular}{llll}
\hline Number of peaks & $q$ & $E(\mathrm{~V})$ & $T(\mathrm{~ns})$ \\
\hline 1 & 0.96 & 2.6 & 43 \\
2 & 0.96 & 2.6268 & 85 \\
4 & 0.97 & 2.8211 & 221 \\
5 & 0.9896 & 2.7809 & 400 \\
6 & 0.9740 & 2.9484 & 354 \\
7 & 0.9786 & 2.9149 & 448 \\
8 & 0.9696 & 2.8948 & 440 \\
9 & 0.9774 & 2.9149 & 553 \\
10 & 0.9728 & 2.8747 & 581 \\
\hline
\end{tabular}

Table 2. Number of peaks in one period of oscillations of $V_{\mathrm{GS}}$ as a function of fractional-order inductors $L_{1}$ and $L_{2}$.

\begin{tabular}{llll}
\hline Number of peaks & $L_{1}(\mu \mathrm{H})$ & $L_{2}(\mu \mathrm{H})$ & $T(\mathrm{~ns})$ \\
\hline 1 & 5 & 4 & 78 \\
2 & 13.704 & 4.332 & 109 \\
4 & 13.704 & 1.908 & 187 \\
6 & 14.384 & 2.22 & 293 \\
8 & 10.3 & 1.644 & 318 \\
\hline
\end{tabular}

Table 3. Number of peaks in one period of oscillations of $V_{\mathrm{GD}}$ as function of the fractional-order capacitors $C_{\mathrm{GS}}$ and $C_{\mathrm{GD}}$.

\begin{tabular}{llll}
\hline Number of peaks & $C_{\mathrm{GS}}(\mathrm{pf})$ & $C_{\mathrm{GD}}(\mathrm{pf})$ & $T(\mathrm{~ns})$ \\
\hline 3 & 3.168 & 6.712 & 148 \\
6 & 2.880 & 5.272 & 273 \\
9 & 3.872 & 5.176 & 455 \\
12 & 2.208 & 4.216 & 483 \\
15 & 3.936 & 5.048 & 761 \\
\hline
\end{tabular}

voltage $V_{\mathrm{GS}}(t)$. Each color represents a number of peaks in a single period of oscillations of $V_{\mathrm{GS}}(t)$. The mathematical description of the observed phenomenon is the spike-adding summarized in Table 1 as the number of peaks in one period of oscillations of $V_{\mathrm{GS}}(t)$ (first column), then the value of the considered period (last column) for a few pairs of parameters $(q, E)$ (middle columns).

Next is Figure 5e the isospike diagram of $L_{2}$ as a function of $L_{1}$, obtained using a palette of five colors, also for the regular oscillations in one period of $V_{\mathrm{GS}}(t)$. Each color represents a number of peaks in one period of oscillations of $V_{\mathrm{GS}}(t)$ too. Except the case of six peaks, the number of peaks in this simulation follow an arithmetic progression of order of $2^{n}$ with $n=0,1,2,3$. Table 2 shows a few couples $\left(L_{1}, L_{2}\right)$ which make it possible to check the number of peaks in one period of the oscillations, as well as the width of this period.

The last isospike diagram (Fig. 5f) presents $C_{\mathrm{GS}}$ as a function of $C_{\mathrm{GD}}$. It has been obtained with a palette of five colors for the regular oscillations in one period of $V_{\mathrm{GD}}(t)$, the voltage between the gate and the drain of the JFET. Each color represents a number of peaks in one period of oscillations of $V_{\mathrm{GD}}(t)$. The number of peaks in this case follows rather an arithmetic progression of order

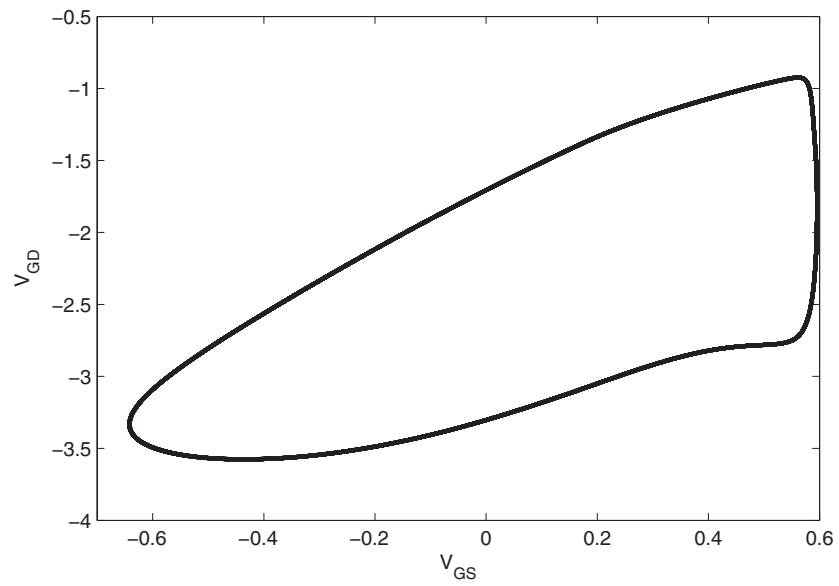

(a)

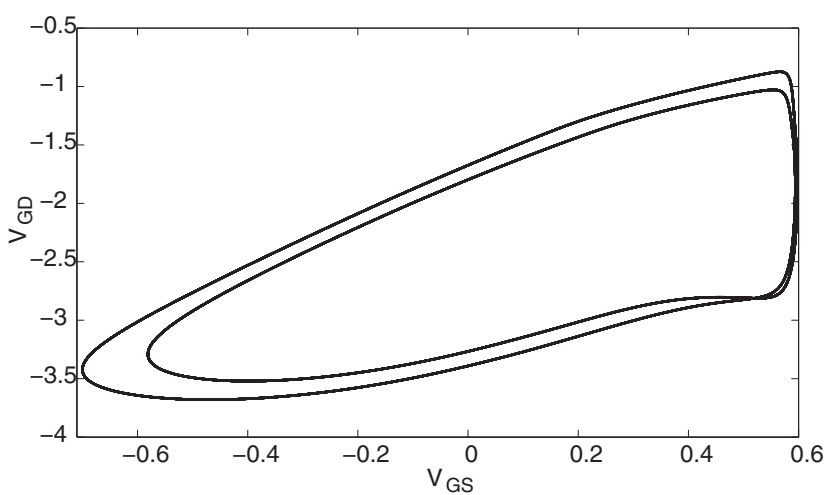

(b)

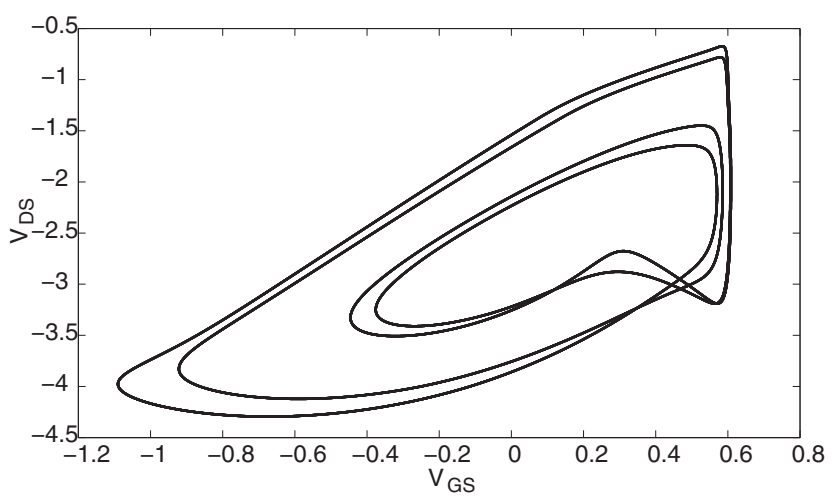

(c)

Fig. 6. Phase portrait $V_{\mathrm{GD}}=f\left(V_{\mathrm{GS}}\right)$ illustrating the number of peaks in one period of $V_{\mathrm{GS}}$ when $q$ and $E$ are fixed and the rest of parameters are chosen as in Section 2.1. The number of peaks here is represented by the number of period in the limit cycle. (a) One peak, (b) two peaks, (c) four peaks, (d) five peaks, (e) six peaks, (f) seven peaks, (g) eight peaks, (h) nine peaks, (i) ten peaks.

of $3 n$ with $n=1,2,3,4,5$. Table 3 shows a few couples $\left(C_{\mathrm{GS}}, C_{\mathrm{GD}}\right)$ which make it possible to check the number of peaks in one period of oscillations, as well as the width of this period.

On these three Isospike diagrams (Figs. 5d-5f), the black color materialize the region of chaotic attractors, which coincides well with the Lyapunov diagrams shown in Figures $5 \mathrm{a}-5 \mathrm{c}$. 


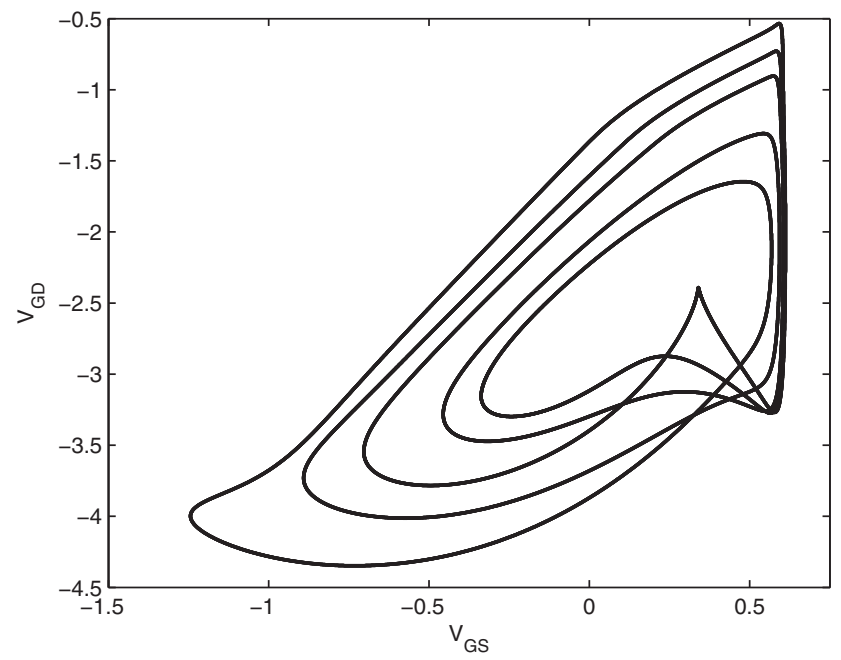

(d)

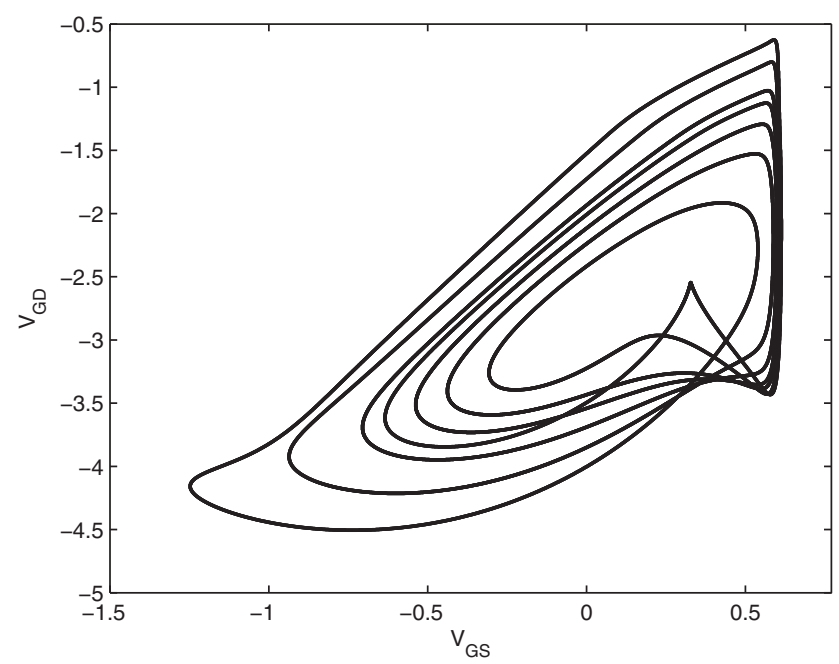

(f)

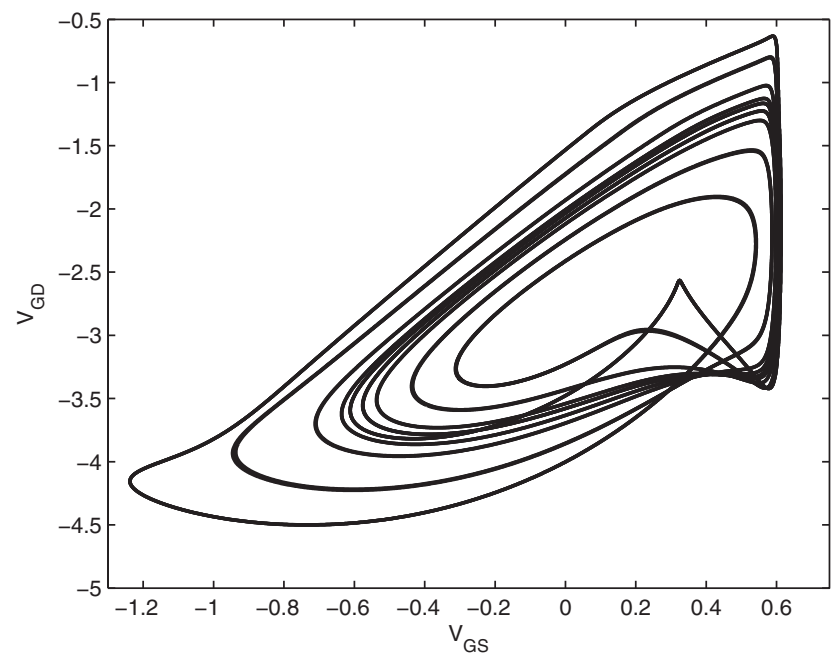

(h)

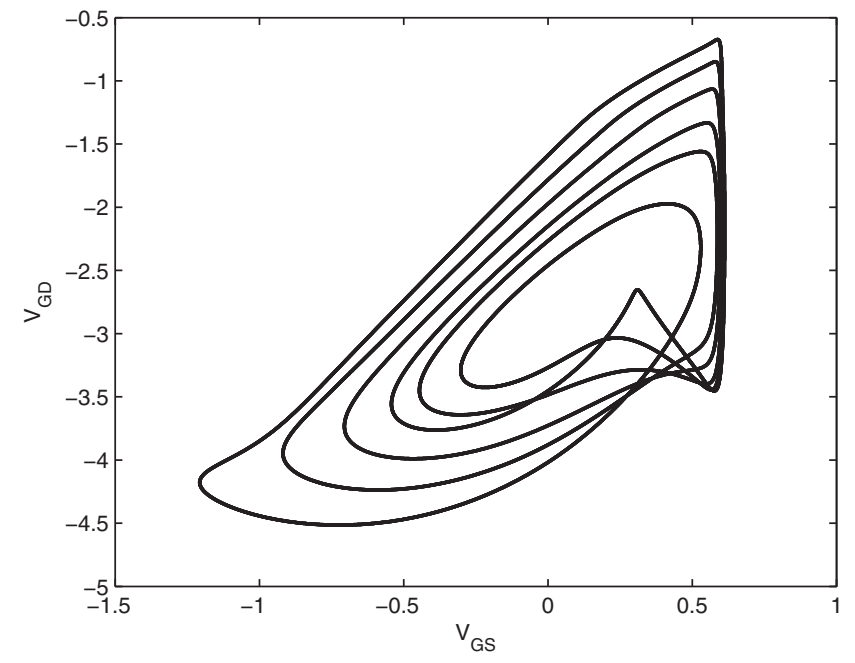

(e)

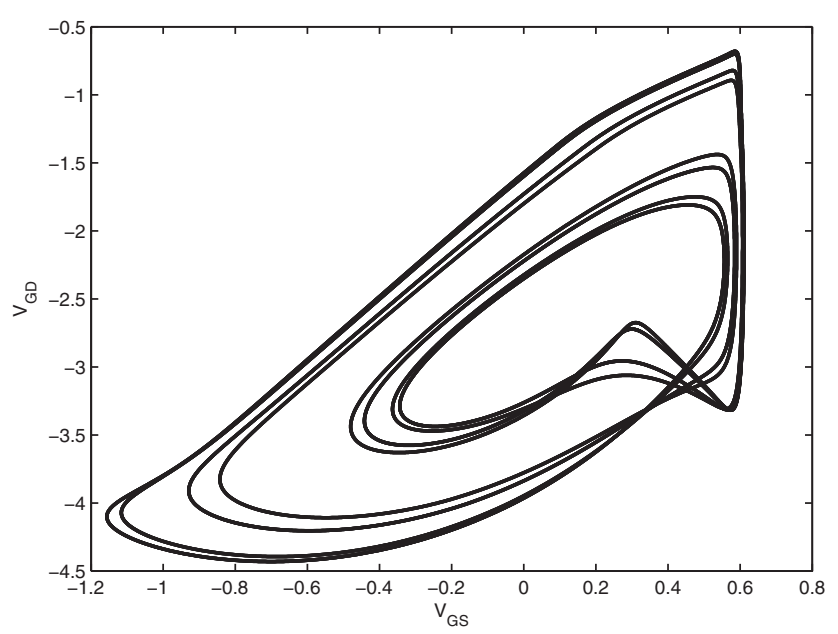

(g)

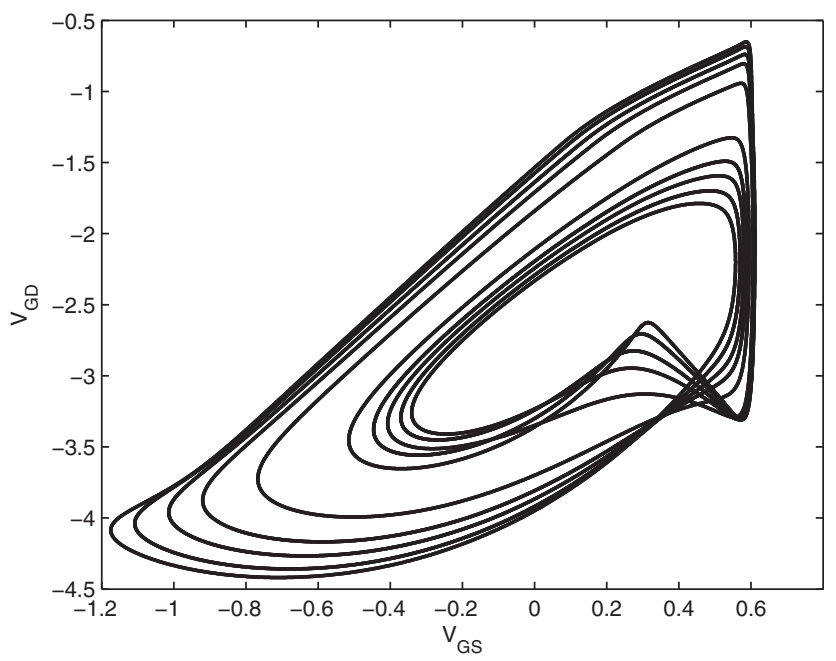

(i)

Fig. 6. (Continued) 


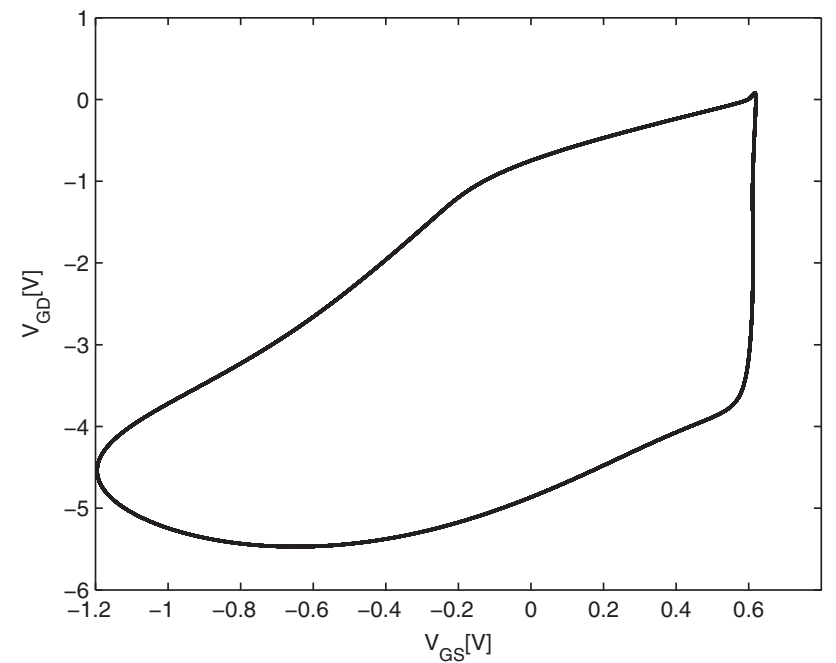

(a)

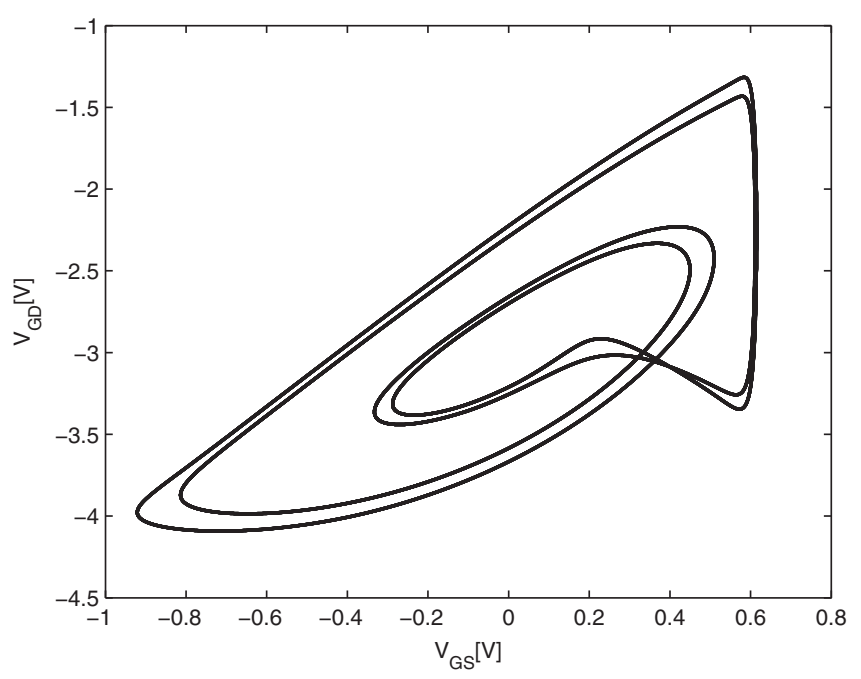

(c)

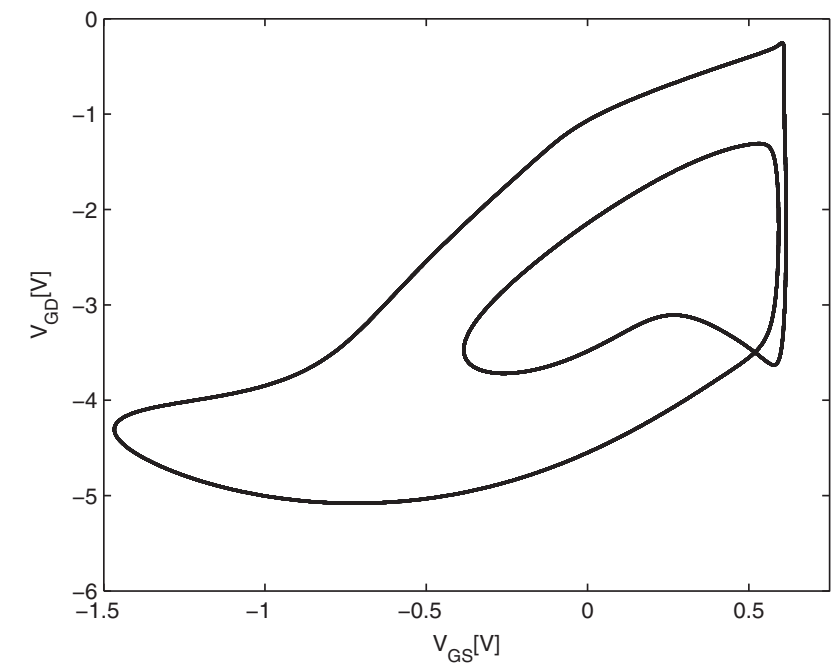

(b)

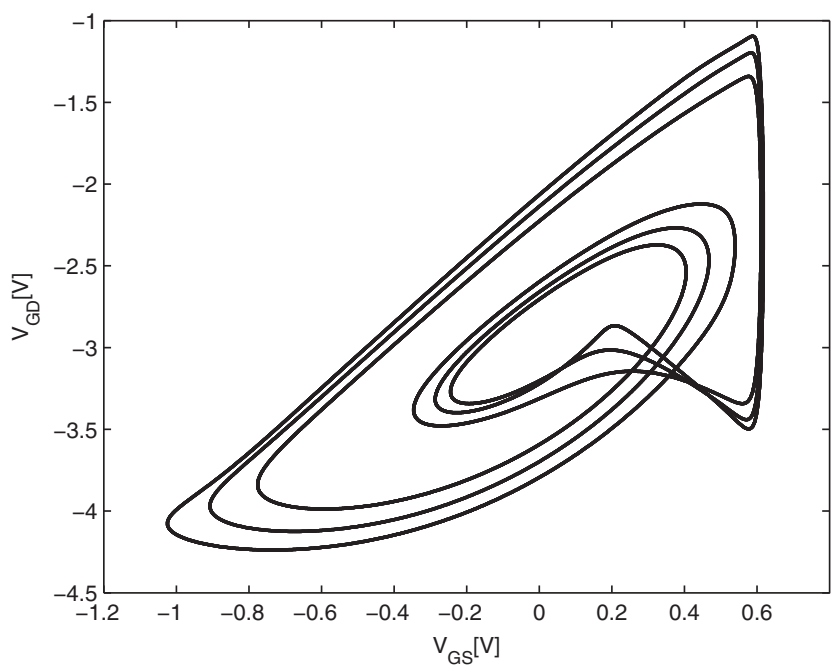

(d)

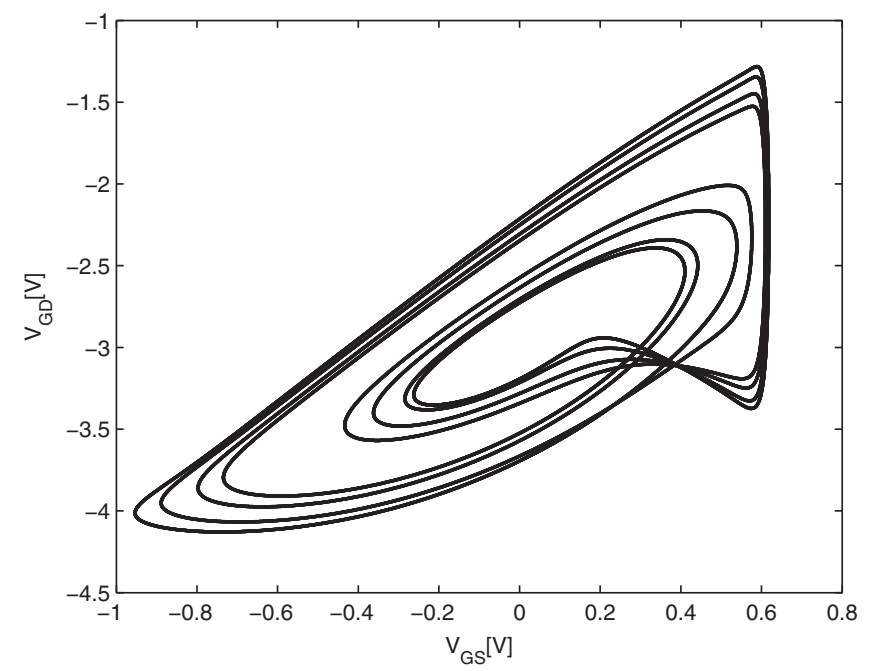

(e)

Fig. 7. Phase portrait $V_{\mathrm{GD}}=f\left(V_{\mathrm{GS}}\right)$ illustrating the number of peaks in one period of $V_{\mathrm{GS}}$ when $L_{1}$ and $L_{2}$ are fixed and $q=0.98$ and $E=3 \mathrm{~V}$. The rest of parameters are chosen as in Section 2.1. The number of peaks here is represented by the number of period in the limit cycle. (a) One peak, (b) two peaks, (c) four peaks, (d) six peaks, (e) eight peaks. 

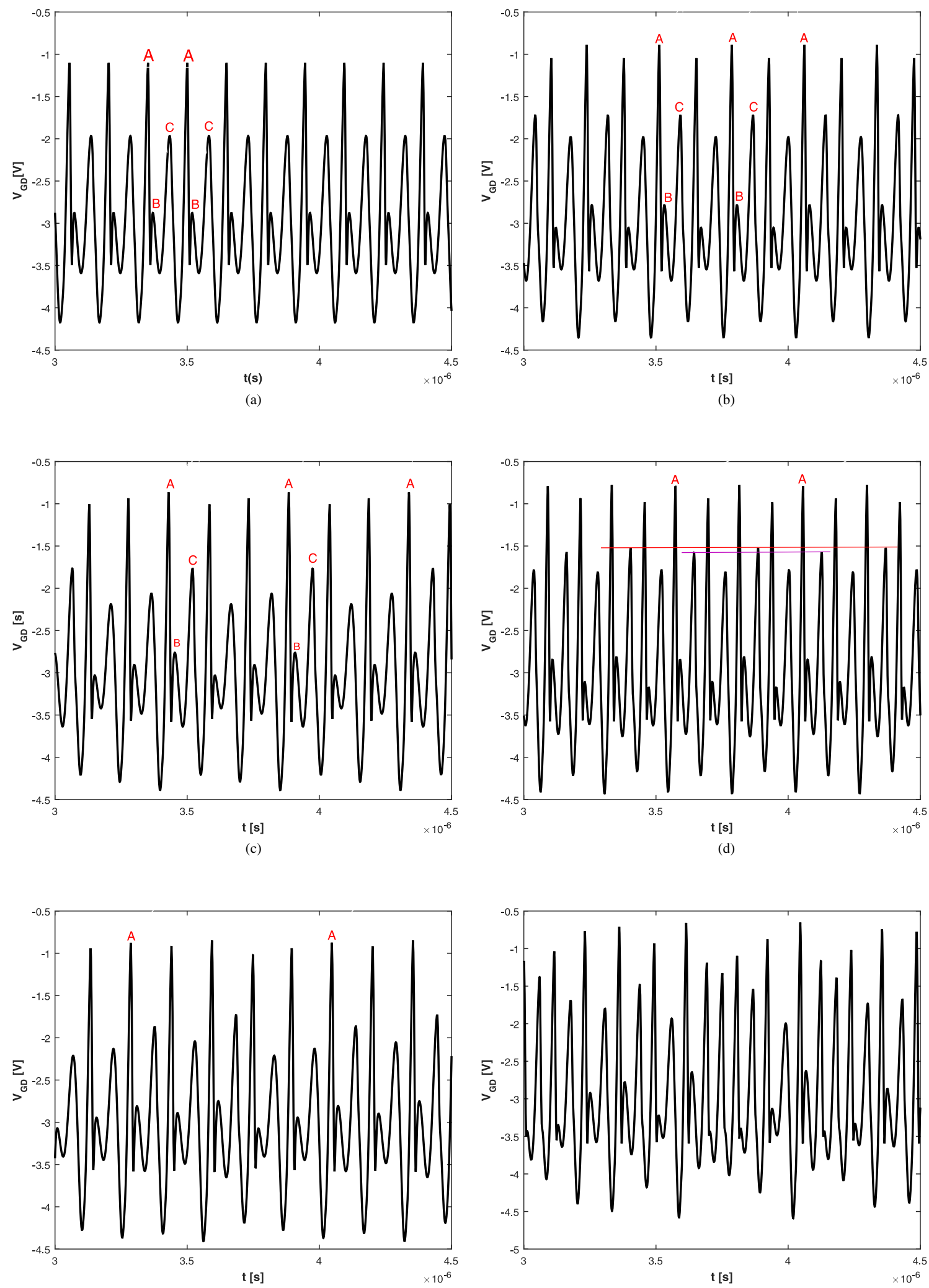

(d)

(e)

Fig. 8. Time serie $V_{\mathrm{GD}}(t)$ illustrating the number of peaks in one period of $V_{\mathrm{GD}}$ when $C_{\mathrm{GS}}$ and $C_{\mathrm{GD}}$ are fixed and $q=0.98$ and $E=3 \mathrm{~V}$. The rest of parameters are chosen as in Section 2.1. The number of peaks in one period of $V_{\mathrm{GD}}(t)$ is represented by the number of maxima in this period. (a) Three peaks, (b) six peaks, (c) nine peaks, (d) twelve peaks, (e) fifteen peaks, (f) chaos. 


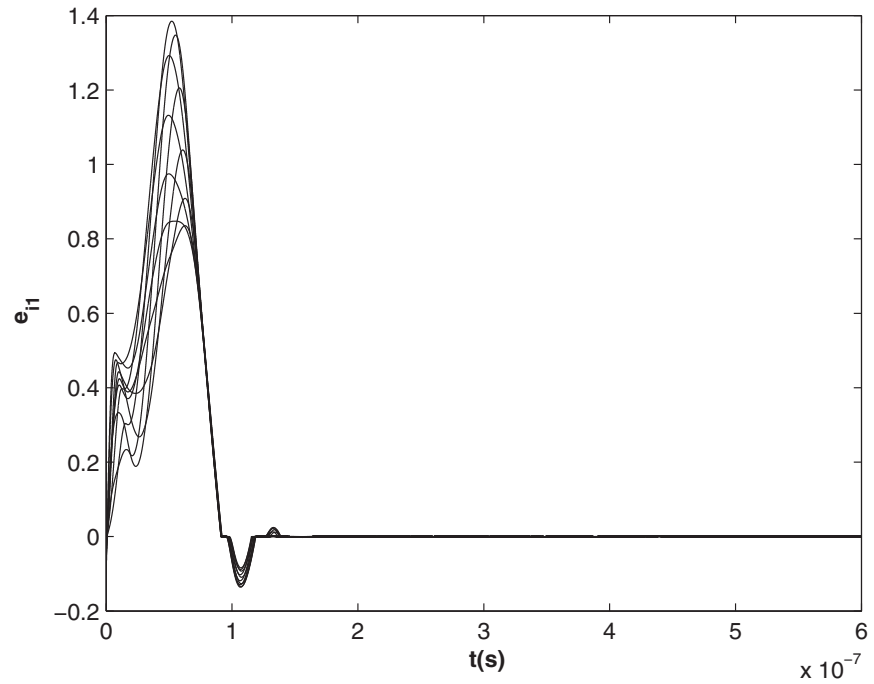

(a)

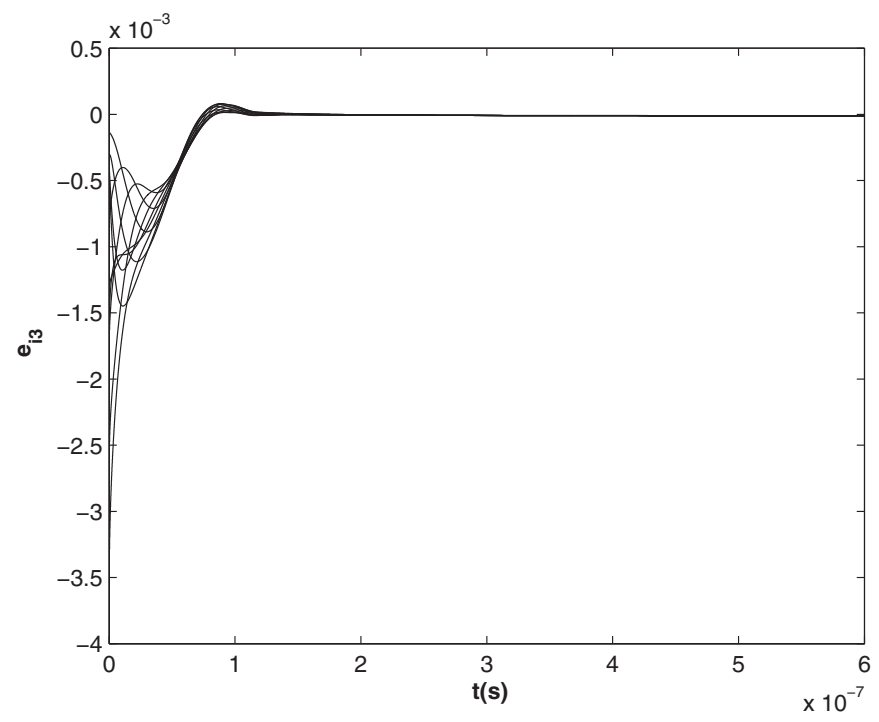

(c)

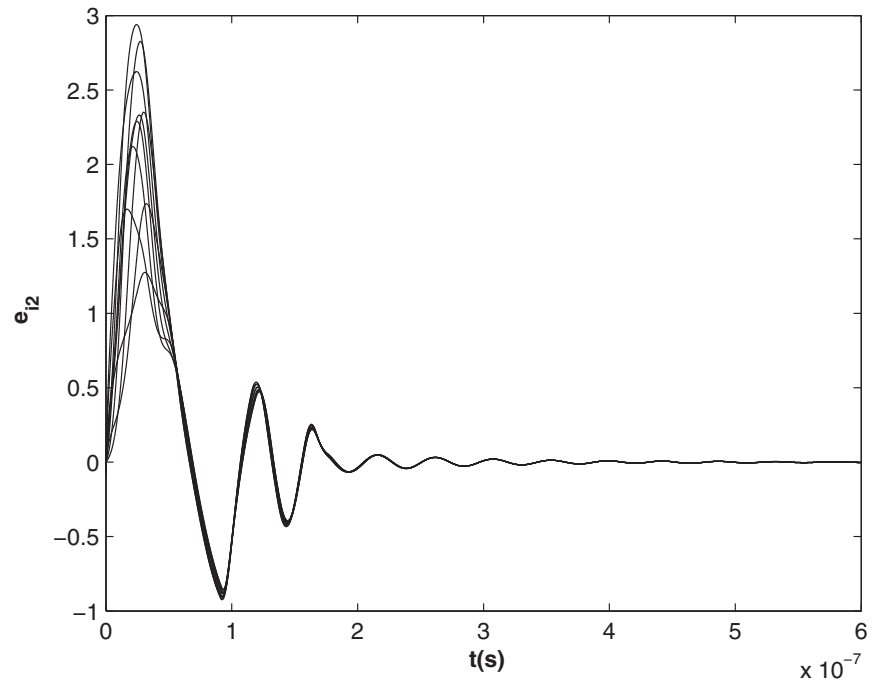

(b)

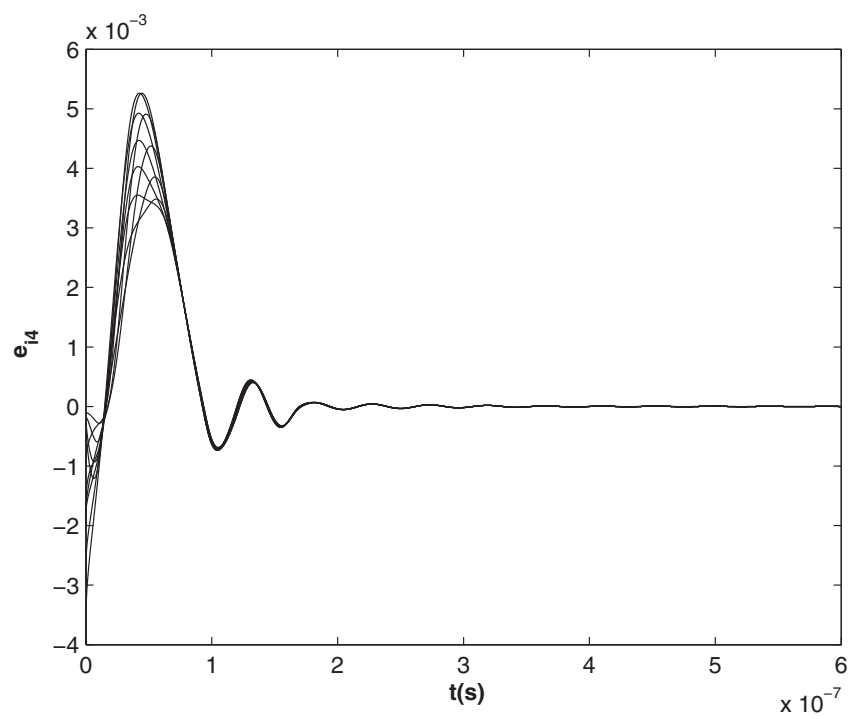

(d)

Fig. 9. Synchronization errors of the complex dynamical networks with an ideal channel.

Figures 6-8 are plotted respectively with respect to Tables 1-3. The letters A, B, C on the Figure 8 enable to count the number of identical peaks in one period of oscillations.

\section{Adaptive sliding mode synchronization and phase transition in a fractional-order complex networks.}

\subsection{Preliminaries}

We consider the drive-response dynamical fractionalorder complex networks with identical nodes:

Master:

$$
\left\{\begin{array}{l}
\frac{d^{q} x^{m}}{d t^{q}}=A x^{m}+B f\left(x^{m}\right)+D g\left(x^{m}\right)+R \\
w=C x^{m}
\end{array}\right.
$$

Slave:

$$
\begin{aligned}
\frac{d^{q} x_{i}^{s}}{d t^{q}}= & A x_{i}^{s}+B f\left(x_{i}^{s}\right)+D g\left(x_{i}^{s}\right)+R \\
& +c \sum_{j=1}^{N} h_{i j} \Gamma x_{i}^{s}+u_{i}, \quad i=1,2, \ldots, N,
\end{aligned}
$$

where the superscripts $m$ and $s$ stand for the master system and slave-networks system respectively. $x^{m}=$ $\left(x_{1}^{m}, x_{2}^{m}, \ldots, x_{n}^{m}\right)^{T} \in R^{n}$, denotes the state vector of the master system, $f$ and $g$ are a continuously differentiable nonlinear functions, $A, B, D, R$, and $C$ are the matrices, $w \in R$ the output. In equation (9), $x_{i}^{s}=$ $\left(x_{i 1}^{s}, x_{i 2}^{s}, \ldots, x_{i n}^{s}\right)^{T} \in R^{n}$ denotes the state vector of the ith node, of the $n$ dimensional dynamical system. $u_{i} \in R^{n}$ 


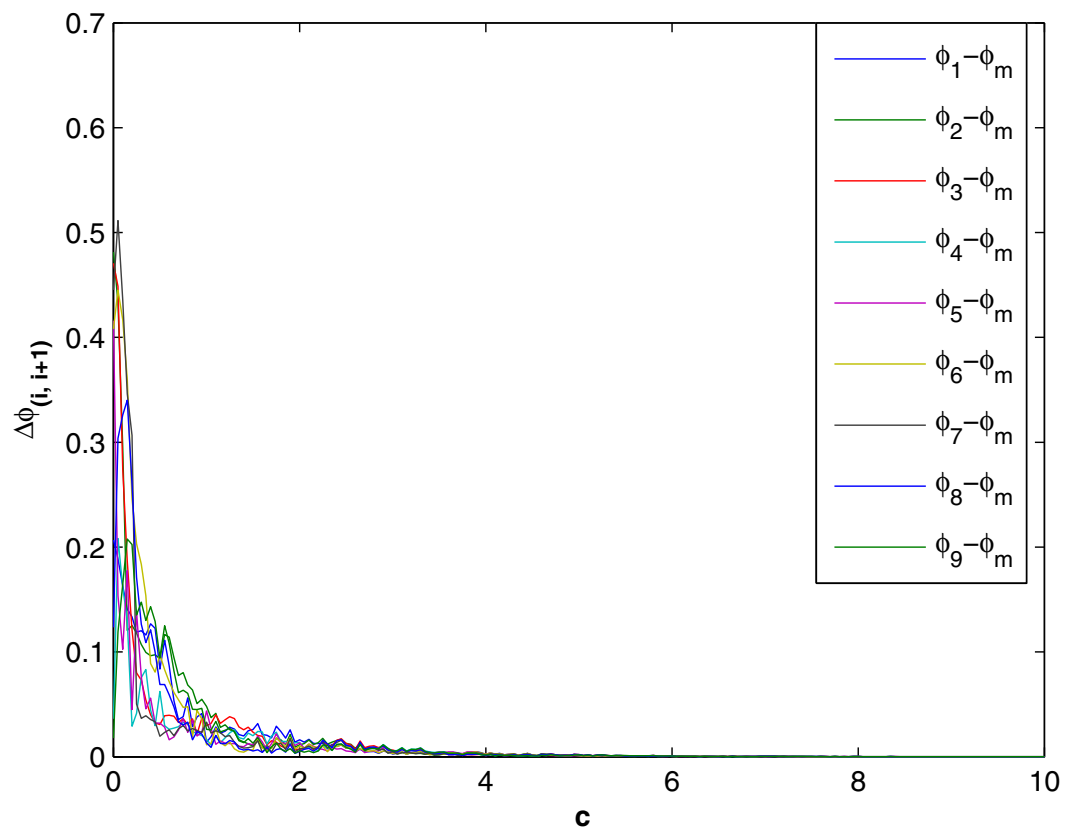

(a)

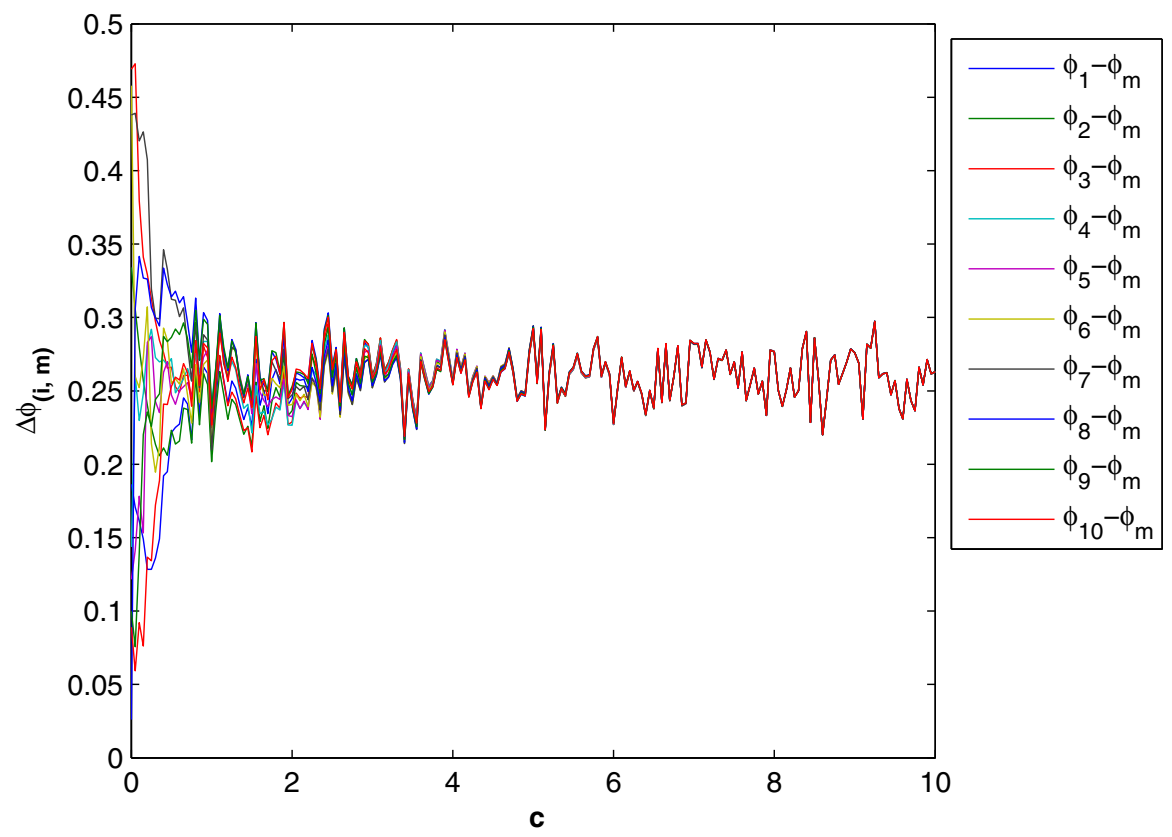

(b)

Fig. 10. (a) Phase difference $\left(\Delta \phi_{i, i+1}\right)$ between any two oscillators of the complex dynamical networks as a function of the coupling strength $c$ for $k=0.6$ with an ideal channel. (b) Phase difference $\left(\Delta \phi_{i, m}\right)$ between one oscillator of the complex dynamical networks and the master oscillator as a function of the coupling strength $c$ for $k=0.6$ with an ideal channel.

is the control input, $\Gamma$ is the inner-coupling term which link the coupled variables in networks. The constant $c>0$ is the coupling strength. $H=\left(h_{i j}\right) \in R^{N \times N}$ is the coupling configuration matrix representing the topological structure of each of the two nodes, in which $h_{i j}$ is defined as follows: if there is a connection between the $i$ th node and the $j$ th node, $(j \neq i)$ then $h_{i j}=h_{j i}=1$; otherwise, $h_{i j}=h_{j i}=0(j \neq i)$, and the diagonal elements of matrix $H$ are defined by:

$$
h_{i i}=-\sum_{\substack{j=1 \\ j \neq i}}^{N} h_{i j}=-\sum_{\substack{j=1 \\ j \neq i}}^{N} h_{j i}, \quad i=1,2, \ldots, N
$$

Definition 1: if $\lim _{t \rightarrow \infty}\left\|e_{i}(t)\right\|=\lim _{t \rightarrow \infty}\left\|x_{i}^{s}(t)-x^{m}(t)\right\|=0$ $(i=1,2, \ldots, N)$ the dynamical network model with identical nodes is achieved synchronization. 


$$
\left\{\begin{array}{l}
u_{i}=K B\left(w(t)-C x_{i}^{s}(t)\right)+G_{i} B \operatorname{sign}\left(w(t)-C x_{i}^{s}(t)\right) \\
\frac{d^{q} G_{i}}{d t^{q}}=d_{i}\left\|\left(w(t)-C x_{i}^{s}(t)\right)\right\|
\end{array} \quad(i=1,2, \ldots, N)\right.
$$

\subsection{Main results}

In this subsection, we propose a novel adaptive control scheme including the sliding mode controller, for the synchronization in complex networks, the adaptive sliding mode controllers and the updating laws are defined as:

\section{See equation (11) above}

where $K$ is a positive constant. The errors equations can be obtained as follows:

$$
\begin{aligned}
\frac{d^{q} e_{i}}{d t^{q}}= & A e_{i}+B\left(f\left(x_{i}^{s}\right)-f\left(x^{m}\right)\right)+D\left(g\left(x_{i}^{s}\right)-g\left(x^{m}\right)\right) \\
& +c \sum_{j=1}^{N} h_{i j} \Gamma e_{j}-K B C e_{i}(t)-G_{i} B \operatorname{sign}\left(C e_{i}(t)\right), \\
& i=1,2, \ldots, N .
\end{aligned}
$$

Remark 1: the choice of matrix $B$ allows us to reduce the number of controllers which ensure the synchronization between one node of dynamical network and the target oscillator.

Assumption 1. The nonlinear functions vectors $f(x)$ and $g(x)$ with $f(0)=0$ and $g(0)=0$ satisfy the Lipschitz condition, that is, there always exists two positive scalars $\eta$ and $\xi$ respectively such that: $\|f(x)-f(\bar{x})\| \leq$ $\eta\|x-\bar{x}\|$ and $\|g(x)-g(\bar{x})\| \leq \xi\|x-\bar{x}\|$.

Assumption 2. The errors matrixes are norm-bounded. It means that there exist two positive constants $\zeta$ and $\theta$ large enough such that: $\left\|D^{T} P e_{i}\right\|=\zeta\left\|B^{T} P e_{i}\right\| \leq \theta$ $\leq \infty$.

Assumption 3. There exists a matrix $L$ and two positive defined and symmetrical matrixes $P$ and $Q$ satisfying the following conditions:

$$
\begin{gathered}
P(A-L C)=-\left(\frac{Q+Q^{T}}{2}\right) \\
B^{T} P=C .
\end{gathered}
$$

Remark 2: for the control design purpose the Lipschitz constants $\eta$ and $\xi$ are normally required to be known. In practice, it is not straightforward to obtain the precise value of $\eta$ and $\xi$. However, it is well known that for real physical systems, the nonlinear functions are always bounded [53]. Moreover quadratic and cubic functions are locally Lipschitz and the Lipschitz constant can be well approximated by the bound on the 2-norm of the Jacobian of the system. The adaptive observer to be designed in the sequel is only guaranteed to be globally convergent in a region of stability depending on $\eta$ and $\xi$.

The error system described by equation (12) can be rewritten as:

$$
\begin{aligned}
\frac{d^{q} e_{i}}{d t^{q}}= & (A-L C) e_{i}+B\left(f\left(x_{i}^{s}\right)-f\left(x^{m}\right)\right) \\
& +D\left(g\left(x_{i}^{s}\right)-g\left(x^{m}\right)\right)+c \sum_{j=1}^{N} h_{i j} \Gamma e_{j}-K B C e_{i}(t) \\
& -G_{i} B \operatorname{sign}\left(C e_{i}(t)\right)+L C e_{i}, \quad i=1,2, \ldots, N,
\end{aligned}
$$

Lemma 1. [61] Let $e(t)=0$ be an equilibrium point for the non autonomous fractional-order system (15). Let us assume that there exists a continuous function $V(e(t), t)$ such that:

- $V(e(t), t)$ is positive definite.

$-{ }_{t_{0}}^{C} D_{t}^{q} V(e(t), t)$ with $q \in(0,1)$ is negative semidefinite, the the origin of system (15) is Lyapunov stable.

Lemma 2. [61] Let $e(t) \in R^{n}$ be a vector of differentiable functions. Then, for any time instant $t \geq t_{0}, q \in(0,1)$ the following relationship holds

$$
\frac{1}{2}_{2}^{t_{0}} D_{t}^{q}\left(e^{T}(t) P e(t)\right) \leq e^{T}(t) P_{t_{0}}^{C} D_{t}^{q} e(t)
$$

where $P \in R^{n * n}$ is a constant, square, symmetric and positive definite matrix.

Theorem 2. The complex network (9) are realized adaptive sliding mode synchronization via the controllers (11) if $K>\varepsilon+\mu \lambda \max \left(L^{T} P P L\right)+c \lambda \max (H \otimes \Gamma)$.

\section{Proof.}

Let us propose the following Lyapunov function candidate, which is positive definite:

$$
V(t)=\frac{1}{2}\left(\sum_{i=1}^{N} e_{i}^{T}(t) P e_{i}(t)+\sum_{i=1}^{N} \frac{1}{d_{i}}\left(G_{i}-\tilde{G}_{i}\right)^{T}\left(G_{i}-\tilde{G}_{i}\right)\right),
$$

where $P \in R^{n * n}$ is a constant symmetric and positive definite matrix. 


$$
\begin{aligned}
&{ }_{t_{0}}^{C} D_{t}^{q} V(t) \leq \sum_{i=1}^{N} e_{i}^{T}\left[P(A-L C) e_{i}+P B\left(f\left(x_{i}^{s}\right)-f\left(x^{m}\right)\right)+P D\left(g\left(x_{i}^{s}\right)-g\left(x^{m}\right)\right)-K P B C e_{i}\right] \\
&+\sum_{i=1}^{N} e_{i}^{T}\left[G_{i} P B \operatorname{sign}\left(C e_{i}\right)+P L C e_{i}+c P \sum_{j=1}^{N} h_{i j} \Gamma e_{j}\right]+\sum_{i=1}^{N}\left(G_{i}-\tilde{G}_{i}\right) \frac{1}{d_{i}} \frac{d^{q} G_{i}}{d t^{q}} \\
&+\sum_{i=1}^{N} e_{i}^{T}\left[G_{i} P B \operatorname{sign}\left(C e_{i}\right)+P L C e_{i}+c P \sum_{j=1}^{N} h_{i j} \Gamma e_{j}\right]+\sum_{i=1}^{N}\left(G_{i}-\tilde{G}_{i}\right) \frac{1}{d_{i}} \frac{d^{q} G_{i}}{d t^{q}} \\
&{ }_{t_{0}} D_{t}^{q} V(t) \leq \sum_{i=1}^{N} e_{i}^{T}\left[P(A-L C) e_{i}\right]+\sum_{i=1}^{N}\left\|\eta_{i} e_{i}\right\|\left\|P B e_{i}\right\|+\sum_{i=1}^{N}\left\|\xi_{i} e_{i}\right\|\left\|P D e_{i}\right\|-\sum_{i}^{T} K P B C e_{i} \\
& t_{t_{0}} D_{t}^{q} V(t) \leq \sum_{i=1}^{N} e_{i}^{T}\left(-\frac{Q+Q^{T}}{2}\right) e_{i}+\sum_{i=1}^{N}\left(\frac{\eta_{i}^{2} \theta_{i}}{\varepsilon_{1}}\left\|B^{T} P e_{i}\right\|+\varepsilon_{1}\left\|e_{i}\right\|^{2}+\frac{\xi_{i}^{2} \theta_{i} \zeta_{i}}{\varepsilon_{2}}\left\|B^{T} P e_{i}\right\|\right) \\
&+\sum_{i=1}^{N}\left(-K\left\|e_{i}\right\|^{2}+\varepsilon_{2}\left\|e_{i}\right\|^{2}+\frac{\theta_{i}}{\mu}\left\|B^{T} P e_{i}\right\|+\mu \lambda \max \left(L^{T} P P L\right)\left\|e_{i}\right\|^{2}-G_{i}\left\|B^{T} P e_{i}\right\|+\left(G_{i}-\tilde{G}_{i}\right) \frac{1}{d_{i}} \frac{d^{q} G_{i}}{d t^{q}}\right) \\
&+c \sum_{i=1}^{N} \sum_{j=1}^{N} e_{i}^{T} h_{\mathrm{ij}} \Gamma e_{j}
\end{aligned}
$$

Taking the fractional derivative to expression (17) and applying Lemma 2, it follows that:

${ }_{t_{0}}^{C} D_{t}^{q} V(t) \leq \sum_{i=1}^{N} e_{i}^{T}(t) P \frac{d^{q} e_{i}(t)}{d t^{q}}+\sum_{i=1}^{N}\left(G_{i}-\tilde{G}_{i}\right) \frac{1}{d_{i}} \frac{d^{q} G_{i}}{d t^{q}}$

where $\tilde{G}_{i}$ is defined in the sequel. Taking the time derivative of error system (15) along (18) we have:

See equation (19) above

Using Assumption 1 equation (19) becomes:

See equation (20) above.

Notice that:

$$
\begin{aligned}
& \left(\eta_{i}\left\|B^{T} P e_{i}\right\|-\varepsilon_{1}\left\|e_{i}\right\|\right)^{2} \geq 0 \Rightarrow\left\|B^{T} P e_{i}\right\|\left\|\eta_{i} e_{i}\right\| \\
& \leq \frac{\eta_{i}^{2}}{\varepsilon_{1}}\left\|B^{T} P e_{i}\right\|^{2}+\varepsilon_{1}\left\|e_{i}\right\|^{2}, \\
& \left(\xi_{i}\left\|D^{T} P e_{i}\right\|-\varepsilon_{2}\left\|e_{i}\right\|\right)^{2} \geq 0 \Rightarrow\left\|D^{T} P e_{i}\right\|\left\|\xi_{i} e_{i}\right\| \\
& \leq \frac{\xi_{i}^{2}}{\varepsilon_{2}}\left\|D^{T} P e_{i}\right\|^{2}+\varepsilon_{2}\left\|e_{i}\right\|^{2},
\end{aligned}
$$

also,

$$
\left\|B^{T} P e_{i}\right\|\left\|P L e_{i}\right\| \leq \frac{1}{\mu}\left\|B^{T} P e_{i}\right\|^{2}+\mu\left\|P L e_{i}\right\|^{2}
$$

Moreover,

$$
\left\|P L e_{i}\right\|^{2}=\left\|P L L^{T} P\right\|\left\|e_{i}\right\|^{2} \leq \lambda_{\max }\left(L^{T} P P L\right)\left\|e_{i}\right\|^{2},
$$

where, $\varepsilon_{1}, \quad \varepsilon_{2}$ and $\mu$ are positive scalars and $\lambda_{\max }\left(L^{T} P P L\right)$ is the maximum eigenvalue of the matrix $L^{T} P P L$. Using Assumption 3, equations (21)-(24), we may now write the following inequality:

See equation (25) above.

The Assumption 2 leads us to:

See equation (26) next page.

Thus, by setting:

$$
\tilde{G}_{i}=\frac{\eta_{i}^{2} \theta_{i}}{\varepsilon_{1}}+\frac{\xi_{i}^{2} \zeta_{i} \theta_{i}}{\varepsilon_{2}}+\frac{\theta_{i}}{\mu}
$$

we have:

$$
\text { See equation (28) next page. }
$$

By choosing: $\frac{d^{q} G_{i}}{d t^{q}}=d_{i}\left\|B^{T} P e_{i}\right\|$, and $\varepsilon=\varepsilon_{1}+\varepsilon_{2}$, the inequality (28) becomes:

See equation (29) next page. 


$$
\begin{aligned}
{ }_{t_{0}}^{C} D_{t}^{q} V(t) \leq & \sum_{i=1}^{N} e_{i}^{T}\left(-\frac{Q+Q^{T}}{2}\right) e_{i}+\sum_{i=1}^{N}\left\|B^{T} P e_{i}\right\|\left(\frac{\eta_{i}^{2} \theta_{i}}{\varepsilon_{1}}+\frac{\xi_{i}^{2} \zeta_{i} \theta_{i}}{\varepsilon_{2}}+\frac{\theta_{i}}{\mu}-G_{i}\right) \\
+ & +\sum_{i=1}^{N}\left(-K\left\|e_{i}\right\|^{2}+\varepsilon_{2}\left\|e_{i}\right\|^{2}+\varepsilon_{1}\left\|e_{i}\right\|^{2}+\mu \lambda_{\max }\left(L^{T} P P L\right)\left\|e_{i}\right\|^{2}+\left(G_{i}-\tilde{G}_{i}\right) \frac{1}{d_{i}} \frac{d^{q} G_{i}}{d t^{q}}\right) \\
+c \sum_{i=1}^{N} \sum_{j=1}^{N} e_{i}^{T} h_{i j} \Gamma e_{j} & \\
{ }_{t_{0}}^{C} D_{t}^{q} V(t) \leq & {\left[\left(-\lambda_{\min }\left(\frac{Q+Q^{T}}{2}\right)+\left(\varepsilon_{1}+\varepsilon_{2}\right)+\mu \lambda_{\max }\left(L^{T} P P L\right)-K+c \lambda_{\max }(H \otimes \Gamma)\right)\right]\|e\|^{2} } \\
& +\left(G_{\mathrm{i}}-\tilde{G}_{i}\right)^{2}\left(\frac{1}{d_{i}} \frac{d^{q} G_{i}}{d t^{q}}-\left\|B^{T} P e_{i}\right\|\right) \\
& \\
& {\left[\left(-\lambda_{\min }\left(\frac{Q+Q^{T}}{2}\right)+\varepsilon+\mu \lambda_{\max }\left(L^{T} P P L\right)-K+c \lambda_{\max }(H \otimes \Gamma)\right)\right]\|e(t)\|^{2} }
\end{aligned}
$$

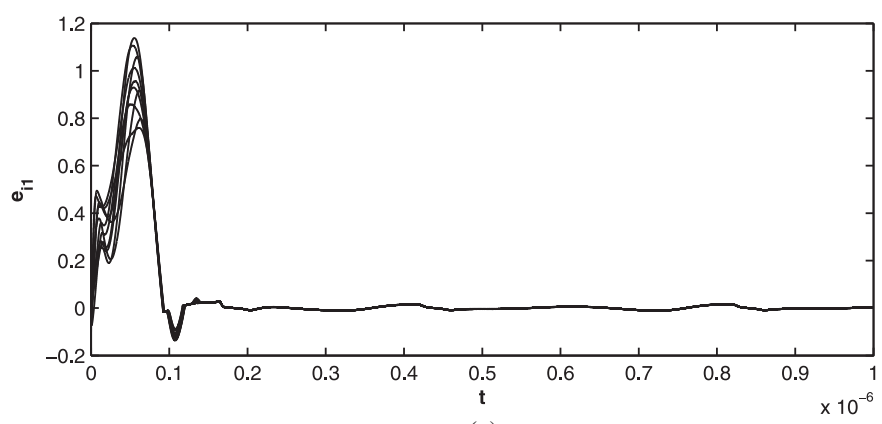

(a)

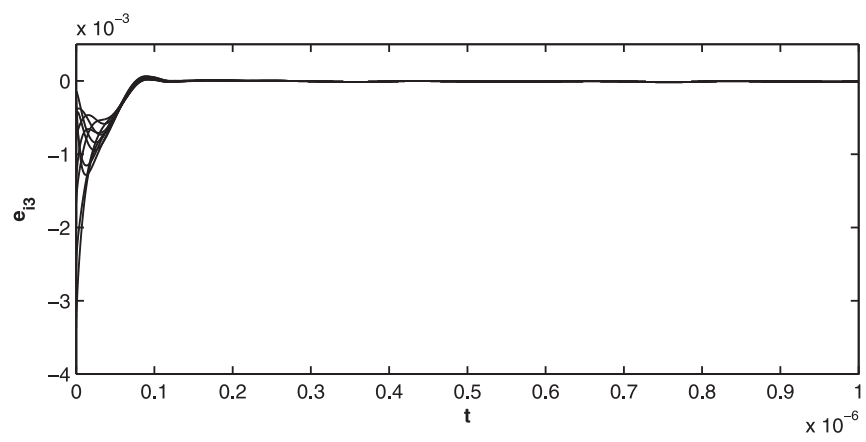

(c)

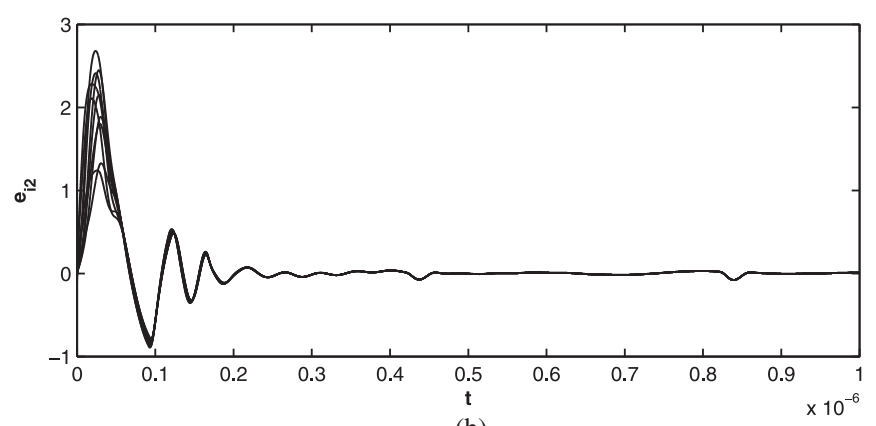

(b)

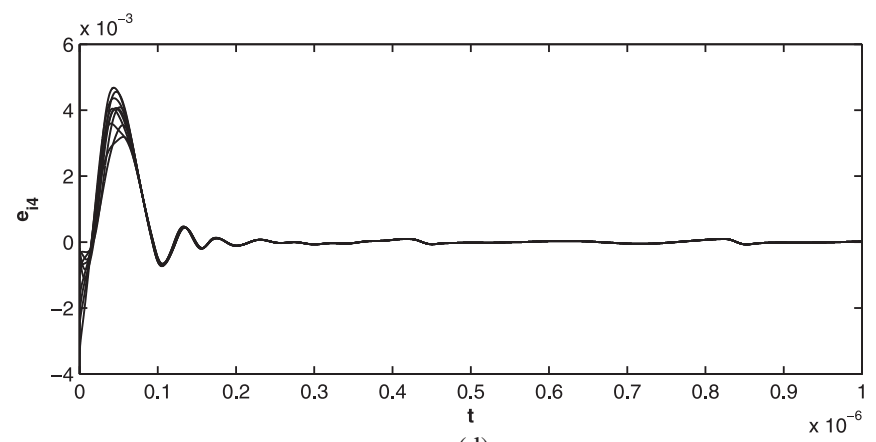

(d)

Fig. 11. Synchronization errors of the complex dynamical networks with a non ideal channel.

Since $\lambda_{\min }\left(\frac{Q+Q^{T}}{2}\right)>0$, it suffices to choose $K>$ $\varepsilon+\mu \lambda_{\max }\left(L^{T} P P L\right)+c \lambda_{\max }(H \otimes \Gamma)$ to turn (29) into ${ }_{t_{0}}^{C} D_{t}^{q} V(t) \leq 0$. According to the fractional-order stability theory (Lemma 1), the system (12) is asymptotically stable. Then the proof is completed.

\subsection{Simulation examples}

The numerical approach for solving fractional-order differential equations is that of Adams-Bashforth-Moulton algorithm for ordinary differential equations. This method is based on a predictor-corrector scheme using the Caputo definition of the fractional differentialequations. The detailed descriptions of this algorithm are available in [1]. For the phase-transition, the phases of the individual oscillators are constructed from the variable $x_{i}(t)$, and the average phase difference $\Delta \phi_{i j}$ between two oscillators is:

$$
\Delta \phi_{i j}=\left\langle\left|\phi_{i}-\phi_{j}\right|\right\rangle
$$

with $\phi_{i}$ defined by:

$$
\phi_{i}=\tan ^{-1}\left(\tilde{x}_{i}(t) / x_{i}(t)\right)
$$




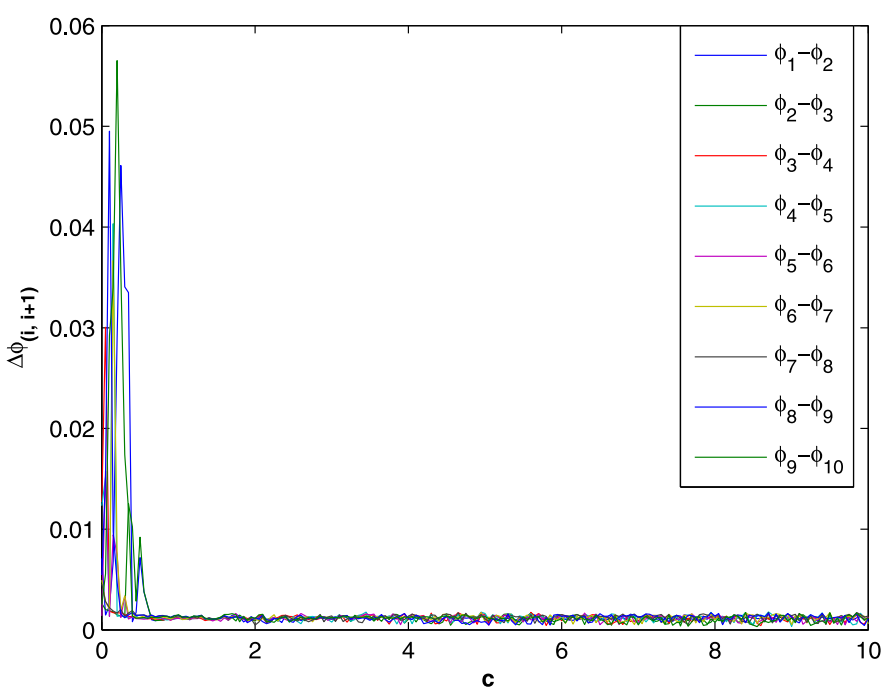

(a)

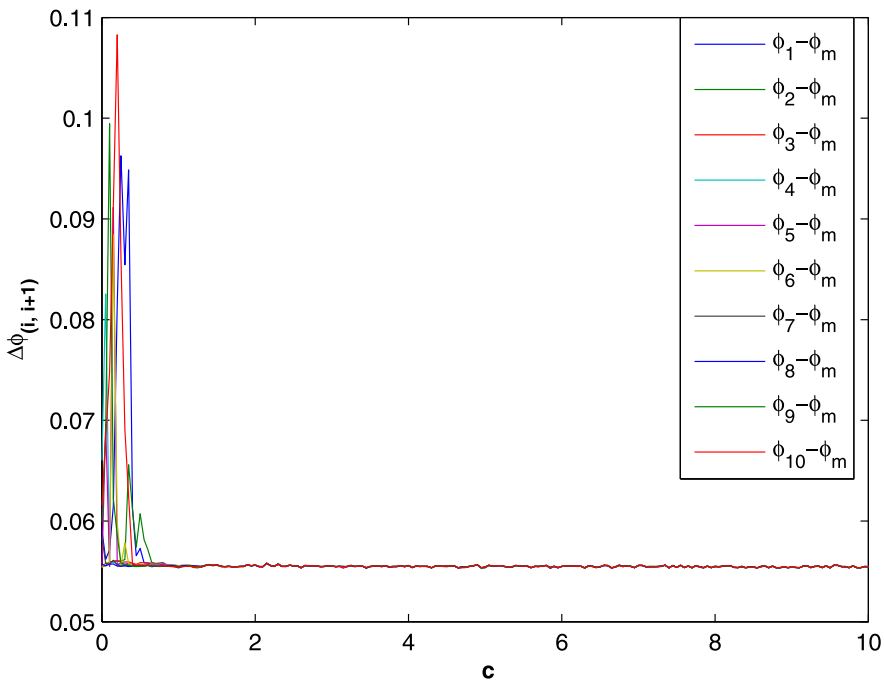

(b)

Fig. 12. (a) Phase difference $\left(\Delta \phi_{i, i+1}\right)$ between any two oscillators of the complex dynamical networks as a function of the coupling strength $c$ for $k=0.6$ and $\tau=1$ ns with a non-ideal channel. (b) Phase difference $\left(\Delta \phi_{i, m}\right)$ between one oscillator of the complex dynamical networks and the master oscillator as a function of the coupling strength $c$ for $k=0.6$ and $\tau=1$ ns with a non ideal channel.

where $\langle$.$\rangle denotes the time average, \tilde{x}_{i}$ is the Hilbert transform of $x_{i}(t)$, the complete procedure is described in [62]. For all numerical simulation concerning the complete and phase synchronization, the parameters values are fixed as follows: $C_{\mathrm{GS}}=3.736 \mathrm{pf}, C_{\mathrm{GD}}=3.35 \mathrm{pf}, L_{1}=24.5 \mu \mathrm{H}$, $L_{2}=4 \mu \mathrm{H}, \quad I_{\mathrm{S}}=33.57 \mathrm{fA}, \quad V_{\mathrm{GSoff}}=-1.409 \mathrm{~V}, g_{m 0}=$ $1.754 \mathrm{mAV}^{-2}, V_{\mathrm{T}}=25 \mathrm{mV}, q=0.96$, and $E=4 \mathrm{~V}$.

\subsubsection{The ideal channel of transmission}

In this section, to demonstrate the effectiveness of the proposed approach, the fractional-order two-component chaotic system as node in the complex network systems is applied to construct the network model with 10 nodes. The different matrices and the different parameters for the systems (8) and (9) are chosen as follows:

$$
\begin{aligned}
& x^{m}=\left(V_{\mathrm{GS}}^{m}, V_{\mathrm{GD}}^{m}, i_{1}^{m}, i_{2}^{m}\right)^{T}, \\
& x_{i}^{s}=\left(V_{\mathrm{GS}_{\mathrm{i}}}^{s}, V_{\mathrm{GD}_{i}}^{s}, i_{1_{i}}^{s}, i_{2_{i}}^{s}\right)^{T} \text {, } \\
& A=\left[\begin{array}{cccc}
0 & 0 & -a_{1} & -a_{1} \\
0 & 0 & 0 & -\alpha a_{1} \\
b_{1} & 0 & 0 & 0 \\
b_{2} & b_{2} & 0 & 0
\end{array}\right] \\
& B=\left[\begin{array}{l}
1 \\
0 \\
0 \\
0
\end{array}\right], \quad D=\left[\begin{array}{c}
-1 \\
\alpha \\
0 \\
0
\end{array}\right], \quad R=\left[\begin{array}{c}
0 \\
0 \\
0 \\
b_{2} E
\end{array}\right] \text {, } \\
& C=\left[\begin{array}{llll}
1 & 0 & 0 & 0
\end{array}\right], \quad f\left(x^{m}\right)=-a_{1} I_{d}\left(V_{\mathrm{GS}}^{m}\right), \\
& f\left(x_{i}^{s}\right)=-a_{1} I_{d}\left(V_{\mathrm{GS}_{i}}^{s}\right), \quad g\left(x^{m}\right)=a_{3} i_{D}\left(V_{\mathrm{GS}}^{m}, V_{\mathrm{GD}}^{m}\right), \\
& g\left(x_{i}^{s}\right)=a_{3} i_{\mathrm{D}}\left(V_{\mathrm{GS}_{i}}^{s}, V_{\mathrm{GD}_{i}}^{s}\right), \quad \Gamma=\operatorname{diag}(1,1,1,1) \text {, } \\
& c=1, \quad K=0.6, \quad d_{i}=0.1, \quad N=10 \\
& H=\left[\begin{array}{cccccccccc}
-1 & 0 & 0 & 0 & 0 & 0 & 0 & 0 & 0 & 1 \\
1 & -1 & 0 & 0 & 0 & 0 & 0 & 0 & 0 & 0 \\
0 & 1 & -1 & 0 & 0 & 0 & 0 & 0 & 0 & 0 \\
0 & 0 & 1 & -1 & 0 & 0 & 0 & 0 & 0 & 0 \\
0 & 0 & 0 & 1 & -1 & 0 & 0 & 0 & 0 & 0 \\
0 & 0 & 0 & 0 & 1 & -1 & 0 & 0 & 0 & 0 \\
0 & 0 & 0 & 0 & 0 & 1 & -1 & 0 & 0 & 0 \\
0 & 0 & 0 & 0 & 0 & 0 & 1 & -1 & 0 & 0 \\
0 & 0 & 0 & 0 & 0 & 0 & 0 & 1 & -1 & 0 \\
0 & 0 & 0 & 0 & 0 & 0 & 0 & 0 & 1 & -1
\end{array}\right]
\end{aligned}
$$

Figure 9 displays the time evolution of the synchronization errors in the network. This figure depicts that the nodes of the network are globally asymptotically stable under the new sliding mode adaptive fractional-order controllers.

Figure 10a shows the phase synchronization between the oscillators of fractional-order complex dynamic network when the constant gain $k$ is fixed at $k=0.6$ and the coupling strength is as control parameter for the phase synchronization. On Figure 10a for $0<c<5$ the average phase difference $\left(\Delta \phi_{i, i+1}=\left\langle\left|\phi_{i}-\phi_{i+1}\right|\right\rangle, i=1,2,3 \ldots 10\right.$ represent superscripts of ten nodes of network and when $i+1=11$ take $i=1$ ) between two oscillators is different from zero what implies that the 10 nodes of fractionalorder complex network are not synchronize in phase. Further increase of the coupling strength gives rise to a regime of phase synchronization of all ten oscillators for $5<c<10$. Figure 10b shows the average phase difference between the isolated node (master) and each of the ten nodes of fractional-order complex network $\left(\Delta \phi_{i, m}=\left\langle\left|\phi_{i}-\phi_{m}\right|\right\rangle, i=1,2,3, \ldots, 10\right.$ and $m$ is superscripts of isolated nodes also called master). In Figure 10b the phase differences are not identical for $0<c<5$ and for $5<c<10$ these phases difference becomes identical but different from zero. This result allows us to conclude that none of the ten nodes is in phase synchronization with the master oscillator. 


\subsubsection{The non ideal channel of transmission}

In this subsection we consider the effect of Gaussian white noise $\Theta=10^{-2} \sqrt{-2 \ln (\mathrm{rand})} \cos (2 \pi$ rand $)$ and the time delays $\tau=1 \mathrm{~ns}$ of channel. This delay is applied on the controller (11) and this equation can be rewritten as follows:

$$
\left\{\begin{array}{l}
u_{i}=K B\left(W(t-\tau)-C x_{i}^{s}(t)\right) \\
+G_{i} B \operatorname{sign}\left(W(t-\tau)-C x_{i}^{s}(t)\right) \\
\frac{d^{q} G_{i}}{d t^{q}}=d_{i}\left\|\left(W(t-\tau)-C x_{i}^{s}(t)\right)\right\| \\
W(t-\tau)=w(t-\tau)+\Theta .
\end{array}\right.
$$

The numerical results are given by Figure 11, this figure confirms the robustness and effectiveness of the proposed schemes.

Figure 12 presents firstly the effect of channel of transmission on the phase difference between the different oscillators of fractional-order complex network (Fig. 12a) and secondly, the phase difference between each oscillator of fractional-order complex network with the master oscillator (Fig. 12b). We can see that the phase differences are identical when the coupling strength takes the value $c=1$ until $c=10$. These values of phase difference are not equal to zero but are nearly zero, indicating complete phase synchronization [62]. By comparison to the previous case we can conclude that the presence of the channel of transmission minimizes the phase difference but never annuls it completely.

\section{Conclusion}

In conclusion, we have studied the fractional-order version of a two-component circuit operating at high frequency using the analytic and numerical methods. The numerical methods have given very interesting results, for example we successfully obtained the range of control parameter which are the bias voltage source and fractional-order, for which the fractional-order two component circuit exhibits the chaotic behavior. The critical values of control parameters for which the Hopf bifurcation arises in the system are given. One of the contributions of this work was the study of the stability of fractional-order two-component circuit by the Lyapunov diagrams and the Isospike diagram. Our diagrams show precisely where the number of spikes changes as a function of control parameters used to count them. In our diagrams, we observe that the number of spikes in one period of oscillations increases following a recently reported non chaos mediated spike adding mechanism. These results show us the richness of the dynamics of the fractional-order two-component circuit. The analytic result allows us to build the novel robust adaptive sliding mode controller for the synchronization of a fractional-order complex network, the application to the case of fractional-order two component circuit show the effectiveness of the proposed synchronization technique as well as the existence of phase-transition in the fractional-order complex network.

\section{Author contribution statement}

Conceptualization: RK and RT; formal analysis: RK and AKST (Hopf bifurcation part), RT and RK (stability part), RK, AKST, AF (adaptive complex network synchronization); methodology: RK, RT and GL; software: RK and RT (isospike program adapted for the fractional order system); validation: RT, GL, and CL; visualization: $\mathrm{AF}$ and CL; writing original draft: RK, RT and GL.

Open Access This is an open access article distributed under the terms of the Creative Commons Attribution License (http://creativecommons.org/licenses/by/4.0), which permits unrestricted use, distribution, and reproduction in any medium, provided the original work is properly cited.

\section{References}

1. I. Podlubny, Fractional Differential Equations (Academic Press, New York, 1999)

2. R. Hilfer, Applications of Fractional Calculus in Physics (World Scientific, Singapore, 2000)

3. H. Sun, A. Abdelwahed, B. Onaral, IEEE Trans. Autom. Control 29, 441 (1984)

4. K. Diethelm, N.J. Ford, A.D. Freed, Nonlinear Dyn. 29, $3(2002)$

5. A. Atangana, I. Koca, Chaos Soliton. Fract. 89, 1 (2016)

6. C.A. Monje, Y. Chen, B.M. Vinagre, D. Xue, V. FeliuBattle, Fractional-order Systems and Controls: Fundamentals and Applications (Springer, London, 2010)

7. I. Petras, Fractional-Order Nonlinear Systems: Modeling, Analysis and Simulations (Springer, Berlin, Heidelberg, 2011)

8. J.C. Wang, J. Electrochem. Soc. 134, 1915 (1987)

9. R.L. Bagley, R.A. Calico, J. Guid. Control Dyn. 14, 304 (1991)

10. B. Ducharne, G. Sebald, D. Guyomar, G. Litak, J. Appl. Phys. 117, 243907 (2015)

11. W.M. Ahmad, R. El-Khazali, Chaos Soliton. Fract. 33, 1367 (2007)

12. L. Song, S. Xu, J. Yang, Commun. Nonlinear Sci. Numer. Simul. 15, 616 (2010)

13. E. Ahmed, A.S. Elgazzar, Physica A 379, 607 (2007)

14. B.S.T. Alkahtani, A. Atangana, Entropy 18, 100 (2016)

15. H.M. Baskonus, Z. Hammouch, H. Bulut, Chaos in the fractional order logistic delay system: Circuit realization and synchronization, AIP Conf. Proc. 1738, 290005 (2016)

16. H.M. Baskonus, T. Mekkaoui, Z. Hammouch, H. Bulut, Entropy 17, 5771 (2015)

17. R. Kengne, R. Tchitnga, S. Mabekou, B.R. Wafo Tekam, G.B. Soh, A. Fomethe, Chaos Soliton. Fract. 111, 6 (2018)

18. R. Kengne, R. Tchitnga, A. Mezatio, A. Fomethe, G. Litak, Eur. Phys. J. B 90, 88 (2017)

19. L.O. Chua, M. Komuro, T. Matsumoto, IEEE Trans. Circ. Syst. I Fundam. Theory Appl. 33, 1073 (1986)

20. T. Hartley, C. Lorenzo, H. Qammer, IEEE Trans. Circ. Syst. I Fundam. Theory Appl. 42, 485 (1995) 
21. C.P. Li, W.H. Deng, D. Xu, Physica A 360, 171 (2006)

22. D. Cafagna, G. Grassi, Int. J. Bifurcat. Chaos 18, 615 (2008)

23. Z. Chaoxia, Y. Simin, Chaos Soliton. Fract. 44, 845 (2011)

24. C. Li, G. Chen, Chaos Soliton. Fract. 22, 540 (2004)

25. R. Kengne, R. Tchitnga, K.A. Tchagna, A. Fomethe, J. Eng. Sci. Technol. Rev. 6, 24 (2013)

26. C. Donato, G. Giuseppe, Nonlinear Dyn. 70, 1185 (2012)

27. R.M. Nguimdo, R. Tchitnga, P. Woafo, Chaos 23, 043122 (2013)

28. R. Tchitnga, H.B. Fotsin, B. Nana, P.H. Louodop, P. Woafo, Chaos Soliton. Fract. 45, 306 (2012)

29. J.G. Freire, J.A.C. Gallas, Chaos Soliton. Fract. 59, 129 (2014)

30. J.G. Freire, R. Meucci, F.T. Arecchi, J.A.C. Gallas, Chaos 25, 097607 (2015)

31. J.G. Freire, C. Cabeza, A.C. Marti, T.P. Oschel, J.A.C. Gallas, Eur. Phys. J. Special Topics 223, 2857 (2014)

32. M.R. Gallas, J.A.C. Gallas, Chaos 25, 064603 (2015)

33. M.R. Gallas, J.A.C. Gallas, Eur. Phys. J. Special Topics 223, 2131 (2014)

34. L. Junges, J.A.C. Gallas, Phys. Lett. A 376, 2109 (2012)

35. L. Junges, T.P. Oschel, J.A.C. Gallas, Eur. Phys. J. D 67, 149 (2013)

36. J. Guan, Optik 127, 4211 (2016)

37. A. Soukkou, A. Boukabou, S. Leulmi, Optik 127, 5070 (2016)

38. H. Xi, Y. Li, X. Huang, Optik 126, 5346 (2015)

39. R. Li, W. Li, Optik 126, 2965 (2015)

40. G.C. Wu, D. Baleanu, L.L. Huang, Appl. Math. Lett. 82, $71(2018)$

41. G.C. Wu, D. Baleanu, H.P. Xie, F.L. Chen, Physica A 460, 374 (2016)

42. W. Xingyuan, Z. Xiaopeng, M. Chao, Nonlinear Dyn. 69, $511(2012)$
43. R. Kengne, R. Tchitnga, K.A. Tchagna, T.A. Nzeusseu, A. Fomethe, J. Chaos 2013, 839038 (2013)

44. W. Sha, Y. Yongguang, D. Miao, Physica A 389, 4981 (2010)

45. D. Hongyue, Chaos Soliton. Fract. 44, 510 (2011)

46. X.J. Wu, H.T. Lu, Chin. Phys. B 19, 070511 (2010)

47. Y. Tang, J. Fang, Commun. Nonlinear Sci. Numer. Simul. 15, 401 (2010)

48. Y. Tang, Z. Wang, J. Fang, Chaos 19, 013112 (2009)

49. Z. Darui, L. Ling, L. Chongxin, Math. Probl. Eng. 2014, 936985 (2014)

50. W.W. Yu, G. Chen, J.H. Lu, Automatica 45, 429 (2009)

51. Y. Chai, L.P. Chen, R.C. Wu, J. Sun, Physica A 391, $5746(2012)$

52. J. Zhou, J.A. Lu, J.H. Lu, Automatica 44, 996 (2008)

53. H. Fotsin, S. Bowong, Chaos Soliton. Fract. 27, 822 (2006)

54. I. Boiko, L. Fridman, R. Iriarte, A. Pisano, E. Usai, Automatica 42, 833 (2006)

55. J. Slotine, W. Li, Applied Nonlinear Control (Prentice Hall, New Jersey, 1991)

56. J. Chen, C. Li, X. Yang, Neurocomputing 313, 324 (2018)

57. J. Fei, C. Lu, J. Franklin Inst. 355, 2369 (2018)

58. S.H. Hosseinnia, R. Ghaderi, A.N. Ranjbar, M. Mahmoudian, S. Momani, Comput. Math. Appl. 59, 1637 (2010)

59. H.L. Li, J. Cao, H. Jiang, A. Alsaedi, J. Franklin Inst. 335, 5771 (2018)

60. A. Syta, G. Litak, S. Lenci, M. Scheffler, Chaos 24, 013107 (2014)

61. M.A. Duarte-Mermoud, N. Aguila-Camacho, J.A. Gallegos, R. Castro-Linares, Commun. Nonlinear Sci. Numer. Simul. 22, 650 (2015)

62. A. Sharma, M.D. Shrimali, A. Prasad, R. Ramaswamy, U. Feudel, Phys. Rev. E 84, 016226 (2011) 